\title{
WEAK EXPLICIT MATCHING FOR LEVEL ZERO DISCRETE SERIES OF UNIT GROUPS OF $p$-ADIC SIMPLE ALGEBRAS
}

\author{
Allan J. Silberger And Ernst-Wilhelm Zink
}

\begin{abstract}
Let $F$ be a p-adic local field and let $A_{i}^{\times}$be the unit group of a central simple $F$-algebra $A_{i}$ of reduced degree $n>1(i=1,2)$. Let $\mathcal{R}^{2}\left(A_{i}^{\times}\right)$denote the set of irreducible discrete series representations of $A_{i}^{\times}$. The "Abstract Matching Theorem" asserts the existence of a bijection, the "Jacquet-Langlands" map, $\mathcal{J L}_{A_{2}, A_{1}}: \mathcal{R}^{2}\left(A_{1}^{\times}\right) \rightarrow \mathcal{R}^{2}\left(A_{2}^{\times}\right)$which, up to known sign, preserves character values for regular elliptic elements. This paper addresses the question of explicitly describing the map $\mathcal{J}$, but only for "level zero" representations. We prove that the restriction $\mathcal{J L}_{A_{2}, A_{1}}: \mathcal{R}_{0}^{2}\left(A_{1}^{\times}\right) \rightarrow \mathcal{R}_{0}^{2}\left(A_{2}^{\times}\right)$is a bijection of level zero discrete series (Proposition 3.2 ) and we give a parameterization of the set of unramified twist classes of level zero discrete series which does not depend upon the algebra $A_{i}$ and is invariant under $\mathcal{J L}_{A_{2}, A_{1}}$ (Theorem 4.1).
\end{abstract}

\section{$\S$ 0. Introduction.}

This paper is the third of a series of papers ([SZ], [GSZ]) in which the authors are working toward an explicit description of the Jacquet-Langlands correspondence for the level zero case.

For the proofs of this paper we depend upon the Abstract Matching Theorem (AMT) (see $\S 0.3$ ), so our results are of the nature, "If AMT is true, then the correspondence has to be this correspondence."

\section{$\S 0.1$ Some Structure and Notation.}

This section will be used throughout and is presented here for easy reference. Our notation will be consistent with that of [GSZ].

Let $F$ be a p-adic local field and $n>1$ an integer. For $d \geq 1$ let $D:=D_{d}$ denote a central $F$-division algebra of index $d$ and let $A:=M_{m}(D)$ a central simple $F$ algebra of reduced degree $n:=d m$.

Let $o:=o_{F}$ denote the ring of integers of $F, \varpi_{F}$ denotes a prime element of $o$, and $\mathfrak{p}_{F}:=\varpi_{F} o$ the maximal ideal of $o$. Let $k:=k_{F}$ denote the residual field of $F$ with $q:=|k|$ its order.

Let $O:=O_{D}$ denote the ring of integers of $D, \varpi:=\varpi_{D}$ a prime element of $O$ such that $\varpi^{d}=\varpi_{F}$, and let $\mathfrak{p}:=\mathfrak{p}_{D}=\varpi O$ be the prime ideal of $O$. We fix a maximal unramified field extension $F_{d} \subset D$ which is normalized by $\varpi$. The residual field $k_{D}:=O / \mathfrak{p}$ is of order $q^{d}$ and may be identified with $k_{d}$, the residual field of $F_{d}$. More generally, for $\ell \geq 1$ we write $F_{\ell}$ for an unramified extension of $F$ of degree $\ell$ and $k_{\ell}$ for a finite field extension of $k$ of degree $\ell$.

We write $X\left(k_{\ell}^{\times}\right)$for the group of multiplicative characters of $k_{\ell}$ and $X_{t}\left(F_{\ell}^{\times}\right)$ for the group of tame multiplicative characters of $F_{\ell}$. Thus $\chi \in X_{t}\left(F_{\ell}^{\times}\right)$has the 
reduction $\bar{\chi} \in X\left(k_{\ell}^{\times}\right)$(see equation (2.3); equation numbers are always within parentheses).

For any ordered partition $s_{1}, \ldots, s_{r}$ of $m$ we call the block diagonal subgroup $M=G L_{s_{1}} \times \cdots \times G L_{s_{r}}$ a standard Levi subgroup of $G L_{m}$ and we call the parabolic subgroup $P=M \ltimes U$ which contains the upper triangular subgroup of $G L_{m}$ a standard parabolic subgroup of $G L_{m}$. When necessary we write $G(R)$ to denote the group of $R$ points of the algebraic group with respect to the ring $R$. Usually we abuse notation to identify algebraic subgroups of $G L_{m}$ with their $D$-points.

For a hereditary order $\mathfrak{A} \subset A=M_{m}(D)$ we write $\mathfrak{P}:=\mathfrak{P}_{\mathfrak{A}}$ for its Jacobson radical. Fix the maximal hereditary order $\mathfrak{A}_{1}=M_{m}(O) \subset A$ and write $\mathfrak{P}_{1}:=$ $\mathfrak{P}_{\mathfrak{A}_{1}}=M_{m}(\mathfrak{p})$. We call $\mathfrak{A}$ standard if $\mathfrak{A} \subset \mathfrak{A}_{1}$ (hence $\left.\mathfrak{P}_{1} \subset \mathfrak{P}_{\mathfrak{A}}\right)$ and if $\left(\mathfrak{A} / \mathfrak{P}_{1}\right)^{\times}$is a standard parabolic subgroup of $G L_{m}\left(k_{D}\right)$. Most of the time we consider standard principal orders $\mathfrak{A}_{r}$, with Jacobson radical $\mathfrak{P}_{r}$, which are determined by the period $r \mid m$ and which are such that $\left(\mathfrak{A}_{r} / \mathfrak{P}_{r}\right)^{\times}$is the standard Levi subgroup $\left[G L_{s}\left(k_{D}\right)\right]^{r}$ of $G L_{m}\left(k_{D}\right)$.

\section{$\S 0.2$ The Category of Level Zero Representations.}

Consider the Bernstein spectrum $\Omega\left(A^{\times}\right)$, the set of $A^{\times}$conjugacy classes $[M, \pi]$ of cuspidal pairs in which $M$ is a standard Levi subgroup of $A^{\times}$and $\pi$ is an irreducible supercuspidal representation of $M$. Each irreducible smooth representation $\Pi$ of $A^{\times}$has a well defined supercuspidal support $\mathcal{C S}(\Pi) \in \Omega\left(A^{\times}\right)$and thus we have a surjective finite to one map

$$
\mathcal{C S}: \operatorname{Irr}\left(A^{\times}\right) \longrightarrow \Omega\left(A^{\times}\right)
$$

The partition of $\Omega\left(A^{\times}\right)$into connected components $\Omega$ pulls back to a partition

$$
\operatorname{Irr}\left(A^{\times}\right)=\bigsqcup_{\Omega} \operatorname{Irr}(\Omega)
$$

where

$$
\operatorname{Irr}(\Omega):=\{\Pi: \mathcal{C S}(\Pi) \in \Omega\}
$$

This partition gives rise to the Bernstein decomposition

$$
\mathcal{M}\left(A^{\times}\right)=\prod_{\Omega} \mathcal{M}(\Omega)
$$

of the category of smooth representations of $A^{\times}$into subcategories $\mathcal{M}(\Omega)$. The set of objects of $\mathcal{M}(\Omega)$ consists of those representations which have all irreducible subquotients in $\operatorname{Irr}(\Omega)$.

Let $\mathcal{C}:=\mathcal{C}(\mathcal{D})$ be a set of representatives for the set of unramified twist classes of irreducible unitary supercuspidal representations of $G L_{s}(D)$ for all $s \geq 1$, and let $\operatorname{Div}^{+}(\mathcal{C})$ be the set of effective divisors on $\mathcal{C}$. Then, with the notation of the second proof of Proposition 2.1,

$$
\mathcal{D}=\sum m_{\pi} \pi \longmapsto \Omega_{\mathcal{D}}=\text { the connected component of }\left[M_{\mathcal{D}}, \pi_{\mathcal{D}}\right] \in \Omega\left(A^{\times}\right)
$$

defines a one-one correspondence between the set of divisors of degree $d(\mathcal{D})=$ $\sum m_{\pi} d(\pi)=m$ and the set of connected components of $\Omega\left(A^{\times}\right)$. 
Let $\mathfrak{A}_{1}, \mathfrak{P}_{1}$ as in $\S_{0.1}$. Then the unit representation $1_{1+\mathfrak{P}_{1}}$ is a type for $A^{\times}$and the subcategory $\mathcal{M}_{0}\left(A^{\times}\right)$of level zero representations of $A^{\times}$has as its set of objects the set of representations $(\Pi, V)$ which are generated by the set of $\left(1+\mathfrak{P}_{1}\right)$-fixed vectors in $V$. It follows either from [BD], Corollaire 3.9, or, more explicitly, from [GSZ], Theorem 5.5 that $\mathcal{M}_{0}\left(A^{\times}\right)$is closed under forming subquotients. Moreover,

$$
\mathcal{M}_{0}\left(A^{\times}\right)=\oplus_{\Omega \text { level zero }} \mathcal{M}(\Omega)
$$

is a finite partial sum of (0.1). (For a general result see [BK2], beginning of Section 4, especially Theorem 4.3.) In the decomposition (0.2) the level zero components may be further represented as $\Omega=\Omega_{\mathcal{D}}$ for divisors $\mathcal{D}=\sum m_{\pi} \pi$ such that all $\pi$ from the support have $\left(1+\mathfrak{P}_{1}\right)$-fixed vectors ([GSZ], Theorem 5.5).

More precisely, consider the residual field $k_{D}$ and the set $\mathcal{C}\left(k_{D}\right)$ of irreducible cuspidal representations of $G L_{s}\left(k_{D}\right)$ for all $s \geq 1$. With respect to the action of $\operatorname{Gal}\left(k_{D} \mid k\right)$ on matrix elements in $G L_{s}\left(k_{D}\right)$, hence on the set of cuspidal representations, let $\overline{\mathcal{C}}\left(k_{D}\right)$ denote the set of $\operatorname{Gal}\left(k_{D} \mid k\right)$ orbits in $\mathcal{C}\left(k_{D}\right)$. Using [GSZ], (27) and Theorem 5.5(ii), we have the bijection

$$
\overline{\mathcal{C}}\left(k_{D}\right) \longleftrightarrow \mathcal{C}_{0} \quad[\sigma] \mapsto \pi_{[\sigma]}
$$

onto the subset of level zero unramified twist class representatives $\mathcal{C}_{0} \subset \mathcal{C}$. From (0.3) we obtain a bijection of divisors

$$
\operatorname{Div}^{+}\left(\overline{\mathcal{C}}\left(k_{D}\right)\right) \longleftrightarrow \operatorname{Div}^{+}\left(\mathcal{C}_{0}\right) \quad \Delta \mapsto \mathcal{D}
$$

We write $\Omega_{\mathcal{D}}=\Omega_{\Delta}$ if $\mathcal{D}$ corresponds to $\Delta$ and note that $(0.2)$ becomes

$$
\mathcal{M}_{0}\left(A^{\times}\right)=\oplus_{\Delta} \mathcal{M}\left(\Omega_{\Delta}\right)
$$

where $\Delta$ runs over the finite set of degree $m$ divisors on $\overline{\mathcal{C}}\left(k_{D}\right)$.

\section{$\S 0.3$ The Abstract Matching Theorem (AMT).}

The Abstract Matching Theorem (AMT) of Deligne/Kazhdan/Vigneras [DKV], Rogawski [Ro], and Badulescu [Ba] asserts that for any two central simple $F$ algebras $A_{1} \cong M_{m_{1}}\left(D_{d_{1}}\right), A_{2} \cong M_{m_{2}}\left(D_{d_{2}}\right)$ of reduced degree $n=d_{1} m_{1}=d_{2} m_{2}$ there exists a bijective mapping, the "Jacquet-Langlands correspondence",

$$
\mathcal{J L}_{A_{2}, A_{1}}: \mathcal{R}^{2}\left(A_{1}^{\times}\right) \longrightarrow \mathcal{R}^{2}\left(A_{2}^{\times}\right) \quad \Pi^{A_{2}}:=\mathcal{J L}_{A_{2}, A_{1}}\left(\Pi^{A_{1}}\right),
$$

where $\mathcal{R}^{2}\left(A_{i}^{\times}\right)$denotes the set of discrete series representations of the unit group $A_{i}^{\times}=G L_{m_{i}}\left(D_{d_{i}}\right)(i=1,2)$, such that the characters $\Theta_{\Pi^{A_{i}}}$ satisfy

$$
(-1)^{m_{1}-1} \Theta_{\Pi^{A_{1}}}(x)=(-1)^{m_{2}-1} \Theta_{\Pi^{A_{2}}}(x)
$$

for all regular elliptic $x$. (We assume an identification of the regular elliptic conjugacy classes among all the unit groups of reduced degree $n$ simple $F$-algebras.)

\section{$\S 0.4$ The Weak Explicit Matching Theorem.}

Let $\mathcal{R}_{0}^{2}\left(A^{\times}\right) \subset \mathcal{R}^{2}\left(A^{\times}\right)$denote the set of level zero discrete series representations and let $\Omega_{\Delta}$ denote a level zero Bernstein component. Proposition 2.8 shows:

(i) $\operatorname{Irr}\left(\Omega_{\Delta}\right) \cap \mathcal{R}_{0}^{2}\left(A^{\times}\right) \neq \emptyset \Longleftrightarrow \Delta=r[\sigma] \in \operatorname{Div}^{+}\left(\overline{\mathcal{C}}\left(k_{D}\right)\right)$ is a simple degree $m$ divisor, i.e. $r d([\sigma])=m$.

(ii) For $\Delta$ a simple divisor as in (i) let $\mathcal{S}_{\Delta}^{A}:=\operatorname{Irr}\left(\Omega_{\Delta}\right) \cap \mathcal{R}_{0}^{2}\left(A^{\times}\right)$. Then $\mathcal{S}_{\Delta}^{A}$ is comprised of precisely one unramified twist class of discrete series representations. 
We say that two representations $\Pi, \Pi^{\prime} \in \mathcal{R}^{2}\left(A^{\times}\right)$are inertially equivalent and write $\Pi \sim \Pi^{\prime}$ if there exists an unramified character $\mu$ of $A^{\times}$such that $\Pi=\Pi^{\prime} \otimes \mu$.

To prove (ii) as well as a certain multiplicity one statement for discrete series representations we reduce to the unramified split case where these facts are well known. For this we use our description of the Hecke algebras of level zero types [GSZ] and establish a reformulation of [BK1](7.7.5) for our context. The Appendix is devoted to these reformulations of Bushnell/Kutzko's work.

Now let $A_{1}, A_{2}$ be as in $\S 0.3$; in particular, assume that $d_{1} m_{1}=d_{2} m_{2}=n$. To give a natural identification of the simple degree $m_{1}$ divisors on $\overline{\mathcal{C}}\left(k_{d_{1}}\right)$ with the simple degree $m_{2}$ divisors on $\overline{\mathcal{C}}\left(k_{d_{2}}\right)$ we use Proposition 1.1 , which constructs out of Green's parameterization of the cuspidal representations of the general linear group over a finite field a natural bijection

$$
\langle\phi\rangle \backslash X\left(k_{n}^{\times}\right) \longleftrightarrow \text { the set of simple degree } m \text { divisors on } \overline{\mathcal{C}}\left(k_{d}\right) \quad[\bar{\chi}] \mapsto \Delta([\bar{\chi}])
$$

for any factorization $n=d m$. The left side consists of the set of $\operatorname{Gal}\left(k_{n} \mid k\right)$ orbits of characters of the multiplicative group $k_{n}^{\times}$. Using (0.6) we may set $\mathcal{S}_{\bar{\chi}}^{A}:=\mathcal{S}_{\Delta([\bar{\chi}])}^{A}$ and this is meaningful for any algebra $A=M_{m}\left(D_{d}\right)$ of reduced degree $n$.

Our main result, the "Weak Explicit Matching Theorem" (Theorem 4.1), asserts that the Jacquet-Langlands correspondence (0.4) restricts to a bijection

$$
\mathcal{J L}_{A_{2}, A_{1}}: \mathcal{R}_{0}^{2}\left(A_{1}^{\times}\right) \longrightarrow \mathcal{R}_{0}^{2}\left(A_{2}^{\times}\right)
$$

of level zero discrete series sets such that $\mathcal{S}_{\bar{\chi}}^{A_{1}}$ maps to $\mathcal{S}_{\bar{\chi}}^{A_{2}}$ for all $[\bar{\chi}] \in\langle\phi\rangle \backslash X\left(k_{n}^{\times}\right)$. We actually prove a slightly finer result: Since AMT implies that $\mathcal{J L}_{A_{2}, A_{1}}$ preserves central characters, we have a partition of $\mathcal{S}_{\bar{\chi}}^{A_{1}}, \mathcal{S}_{\bar{\chi}}^{A_{2}}$ into finite subsets $\mathcal{S}_{\chi}^{A_{1}}, \mathcal{S}_{\chi}^{A_{2}}$, respectively, of cardinality $e:=n / f, f:=|[\bar{\chi}]|$, where $\chi \in X_{t}\left(F_{n}^{\times}\right)$has the reduction $\bar{\chi}$ and $\chi_{F}:=\left.\chi\right|_{F^{\times}}$is the common central character of all the representations in $\mathcal{S}_{\chi}^{A_{1}} \cup \mathcal{S}_{\chi}^{A_{2}}$. We prove that $\mathcal{J}\left(\mathcal{S}_{\chi}^{A_{1}}\right)=\mathcal{S}_{\chi}^{A_{2}}$.

Let us conclude this section by explaining why Theorem 4.1 is called explicit. Let $\bar{\chi} \in\langle\phi\rangle \backslash X\left(k_{n}^{\times}\right)$correspond to the simple degree $m$ divisor $r[\sigma]$ on $\overline{\mathcal{C}}\left(k_{d}\right)(\sigma$ constructed via the correspondence (0.6); cf Proposition 1.1) and let $A=M_{m}\left(D_{d}\right)$. Then we give two explicit characterizations of the representations $\Pi \in \mathcal{S}_{\bar{\chi}}^{A}$, which distinguish this class from all other discrete series representations of $A^{\times}$, each characterization being sufficient to determine the inertial class of $\Pi \in \mathcal{R}_{0}^{2}\left(A^{\times}\right)$:

(a) Let $\mathfrak{A}_{r}$ be the standard principal order of period $r$ in $A$; thus $\mathfrak{A}_{r}^{\times} /\left(1+\mathfrak{P}_{r}\right) \cong$ $\left[G L_{s}\left(k_{d}\right)\right]^{r}(m=r s)$. Then $\Pi \in \mathcal{S}_{\bar{\chi}}^{A}$ if and only if $\Pi$ contains the lift of $\sigma^{\otimes r}$ to an irreducible representation of $\mathfrak{A}_{r}^{\times}$.

(b) Let $M$ be the standard block diagonal Levi subgroup $\left[G L_{s}(D)\right]^{r}$ of $A^{\times}$. Then $\Pi \in \mathcal{S}_{\bar{\chi}}^{A}$ if and only if the supercuspidal support of $\Pi$ is the conjugacy class of an unramified twist of the representation $\pi_{[\sigma]}^{\otimes r}$ of $M$. By [GSZ], Proposition 5.1, the supercuspidal representation $\pi_{[\sigma]}$ of $G L_{s}(D)$ is "explicitly" given by induction from a compact mod center subgroup of $A^{\times}$.

Finally, the "weak explicit matching theorem" is "weak" in the sense that it is explicit only up to the inertial ambiguities which remain to be resolved.

\section{$\S 0.5$ Concerning Some Commutative Diagrams.}

So far we have considered algebras $A \mid F$ of fixed reduced degree $n$. Now we compare $A:=A^{(1)}=M_{m}(D)$ with $A^{(r)}:=M_{m / r}(D)$, where $r \mid m$. 
In the second proof of Proposition 2.1 and in the subsequent Remarks we introduced for any $\Pi \in \mathcal{R}^{2}\left(A^{\times}\right)$the base representation ${ }^{b} \Pi$, which is a unitary supercuspidal representation of $A^{(r)^{\times}}$for some $r \mid m$. The representation ${ }^{b} \Pi$ has the property that the supercuspidal support $\mathcal{C S}(\Pi)$ has a standard representative of the form $\left({ }^{b} \Pi\right)^{\otimes r} \psi$, where $\psi$ is a positive, real-valued character of the standard Levi subgroup $\left[G L_{s}(D)\right]^{r}$. The existence of ${ }^{b} \Pi$ is well known for $D=F$ and can be deduced for other $D$ from AMT (see [DKV]). Because $\Pi \in \mathcal{R}^{2}\left(A^{\times}\right)$is uniquely determined from ${ }^{b} \Pi$, which we prove in Proposition 2.4 only for the case of $\Pi \in \mathcal{R}_{0}^{2}\left(A^{\times}\right)$, we obtain for any $r \mid m$ an injection

$$
\varphi_{D}: \mathcal{R}_{0}^{2}\left(A^{(r)^{\times}}\right) \hookrightarrow \mathcal{R}_{0}^{2}\left(A^{\times}\right),
$$

which is defined uniquely such that $\Pi^{(r)}$ and $\varphi_{D}\left(\Pi^{(r)}\right)$ have the same base representation. For $[\bar{\chi}] \in\langle\phi\rangle \backslash X\left(k_{n}^{\times}\right)$such that $f=|[\bar{\chi}]|$ divides $\frac{n}{r}$ we obtain a unique pull back $\left[\bar{\chi}^{(r)}\right] \in\langle\phi\rangle \backslash X\left(k_{n / r}^{\times}\right)$and $\varphi_{D}$ restricts then to a bijection

$$
\varphi_{D}: \mathcal{S}_{\bar{\chi}^{(r)}}^{A^{(r)}} \stackrel{\sim}{\longrightarrow} \mathcal{S}_{\bar{\chi}}^{A} .
$$

This implies that for $r \mid\left(m_{1}, m_{2}\right)$ and $A_{i}=M_{m_{i}}\left(D_{d_{i}}\right)$ with $n=d_{i} m_{i}(i=1,2)$ the diagram

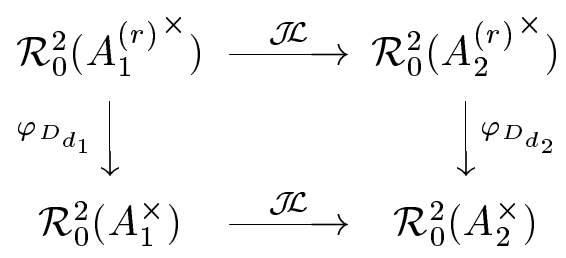

is commutative up to unramified twist. From [DKV], Theorem B.2.b we know that, in fact, (0.7) is commutative.

This suggests comparing the diagrams (0.7) against another set of obviously commutative diagrams. In a future paper, introducing "Langlands parameters", we will define bijections

$$
\bigsqcup_{f \mid n}\langle\phi\rangle \backslash X_{t}\left(F_{f}^{\times}\right)_{\mathrm{reg}} \ni\left[\chi_{f}\right] \longmapsto \Pi_{\chi_{f}}^{A} \in \mathcal{R}_{0}^{2}\left(A^{\times}\right)
$$

such that $\mathcal{J L}_{A^{\prime}, A}\left(\Pi_{\chi_{f}}^{A}\right)=\Pi_{\chi_{f}}^{A^{\prime}}$. The diagrams

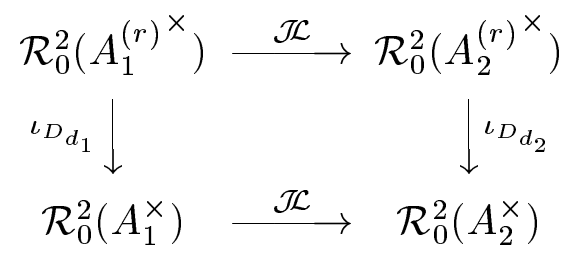

in which

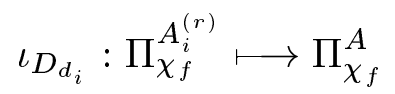

are defined for $f \mid \frac{n}{r}$ and are obviously commutative. When $r \mid\left(m_{1}, m_{2}\right)$, these diagrams can be compared to the corresponding diagrams (0.7). When they can be compared, the diagrams (0.7) and (0.8) agree up to unramified character twist because $\Pi_{\chi_{f}}^{A} \in \mathcal{S}_{\bar{\chi}}^{A}$, where $\chi=\chi_{f} \circ N_{F_{n} \mid F_{f}}$, but in general they are not the same. In fact, the mappings $\varphi_{D_{d_{i}}}$ and $\iota_{D_{d_{i}}}$ differ at most by twists by sign characters which depend upon $n, r, f$. In a future paper we shall make these twists explicit. 
$\S 1$. Parameters for the Set of Level Zero Simple Components of $\Omega\left(A^{\times}\right)$.

The purpose of the following Proposition is to prove (0.6), which gives a natural parameterization for the set of Bernstein components which correspond to level zero simple divisors. Only such components can have level zero discrete series subquotients associated to them.

1.1 Proposition. Let $k_{n} \mid k$ be a degree $n$ extension of finite fields with generating automorphism $\phi$ and let $n:=d m$. Then Green's parameterization of the cuspidal representations of finite general linear groups in terms of orbits of regular characters [Gr] induces a natural bijection

$$
\mathcal{G}_{m}:\langle\phi\rangle \backslash X\left(k_{n}^{\times}\right) \stackrel{\sim}{\longleftrightarrow} \bigsqcup_{s \mid m} G L_{s}\left(k_{d}\right)_{\text {cusp }}^{\wedge} /\langle\phi\rangle
$$

between the set of $\langle\phi\rangle$-orbits in the group of characters $X\left(k_{n}^{\times}\right)$and the set of orbits of cuspidal representations of the groups $G L_{s}\left(k_{d}\right)$ for all $s \mid m$. If $[\bar{\chi}]$ is a character orbit of length $f$ and $f \mid n$, then the corresponding Galois orbit of cuspidal representations $[\sigma]$ consists of representations of $G L_{f^{\prime}}\left(k_{d}\right)\left(f^{\prime}:=f /(d, f)\right)$ and the length of $[\sigma]$ is $(d, f)$. In particular,

$$
|[\bar{\chi}]|=d(\sigma)|[\sigma]|=f^{\prime}(d, f),
$$

$d(\sigma)=f^{\prime}$ being the degree of the divisor $\sigma$.

\section{Remarks.}

(i) For $m=1$ and $d=n$ the correspondence $\mathcal{G}_{1}$ is the identity. For $m=n$ and $d=1$, since there is no Galois action on the right side of (1.1), we see that $\mathcal{G}_{n}$ gives the usual Green's parameterization of the cuspidal representations of $G L_{s}(k)$ for $s \mid n$.

(ii) On the right in (1.1) it would be enough to let $\phi$ be a generator of the Galois group $\operatorname{Gal}\left(k_{d} \mid k\right)$.

Proof. Given a $\langle\phi\rangle$-orbit $[\bar{\chi}] \subset X\left(k_{n}^{\times}\right)$of length $f$ we write $\operatorname{lcm}(d, f)=d f^{\prime}$, and we use the following diagram of finite fields:

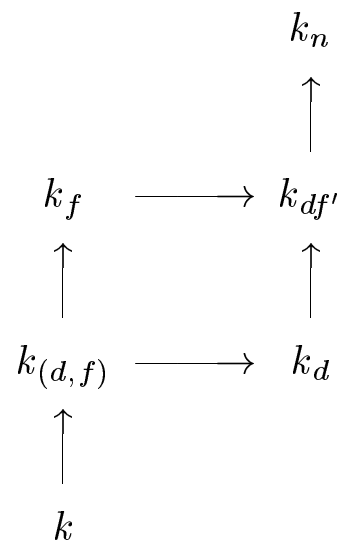

Since $f \mid d f^{\prime}$ and $d f^{\prime} \mid n$, for any $\bar{\chi} \in X\left(k_{n}^{\times}\right)$such that $[\bar{\chi}]=f$, we have a unique $k_{d^{-}}$regular character $\bar{\chi}_{d f^{\prime}} \in X\left(k_{d f^{\prime}}^{\times}\right)$such that $\bar{\chi}_{d f^{\prime}} \circ \mathrm{N}_{k_{n} \mid k_{d f^{\prime}}}=\bar{\chi}$. Therefore, by $[\mathrm{Gr}]$, we also have the unique cuspidal representation $\sigma=\sigma\left(\bar{\chi}_{d f^{\prime}}\right)$ of $\mathrm{GL}_{f^{\prime}}\left(k_{d}\right)$ which has the character values

$$
\theta_{\sigma}(x)=(-1)^{f^{\prime}-1} \sum_{\bar{\eta} \in \operatorname{Gal}\left(k_{d f^{\prime}} \mid k_{d}\right) \cdot \bar{\chi}_{d f^{\prime}}} \bar{\eta}(x)
$$


for $k_{d^{-}}$regular $x \in k_{d f^{\prime}}^{\times}$. Observing that the bijection of Green

$$
\left\langle\phi^{d}\right\rangle \backslash X\left(k_{d f^{\prime}}^{\times}\right)_{k_{d}-\mathrm{reg}} \rightarrow \mathrm{GL}_{f^{\prime}}\left(k_{d}\right)_{\mathrm{cusp}}^{\wedge}
$$

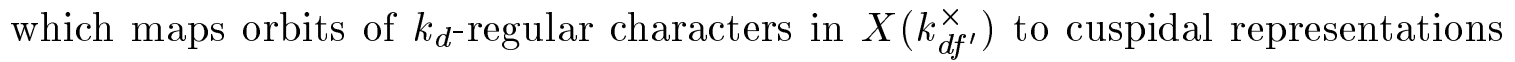
of $G L_{f^{\prime}}\left(k_{d}\right)$, is compatible with the action of $\langle\phi\rangle$ on both sides, we see that the $\langle\phi\rangle$-orbit $\left[\sigma\left(\bar{\chi}_{d f^{\prime}}\right)\right]$ has length $(d, f)$. Conversely, an orbit $[\sigma] \in G L_{s}\left(k_{d}\right)_{\text {cusp }}^{\wedge} /\langle\phi\rangle$ determines an orbit of $k_{d}$-regular characters $\left[\bar{\psi}_{\sigma}\right] \in\langle\phi\rangle \backslash X\left(k_{d s}^{\times}\right)$and a unique orbit $\left[\bar{\chi}_{\sigma}\right] \subset X\left(k_{n}^{\times}\right)$such that $\bar{\chi}_{\sigma}=\bar{\psi}_{\sigma} \circ N_{k_{n} \mid k_{d s}}$. These orbits are of the same length; specifically, we have

$$
\left|\left[\bar{\psi}_{\sigma}\right]\right|=\left|\left[\bar{\chi}_{\sigma}\right]\right|=s|[\sigma]| \text {. }
$$

For $\bar{\chi} \in X\left(k_{n}^{\times}\right)$we write $e:=n / f$ and $e^{\prime}:=(e, m)$. From the equation $d m=e f$ it follows that $m=e^{\prime} f^{\prime}$. Via $\mathcal{G}_{m}$ the character orbit $[\bar{\chi}]$ determines the simple divisor $e^{\prime}[\sigma]$, which has the degree $m$.

Now consider the central simple algebra $A=M_{m}(D), D:=D_{d}$ a central division algebra of index $d$ over $F$. From [GSZ](27) we know how to associate to $[\sigma]$ a unitary supercuspidal representation $\pi_{[\sigma]} \in \mathcal{C}(D)$ such that $d\left(\pi_{[\sigma]}\right)=d([\sigma])$. Thus $[\bar{\chi}]$ determines both a degree $m$ divisor $\mathcal{D}_{m}([\bar{\chi}])=e^{\prime} \pi_{[\sigma]} \in \operatorname{Div}(\mathcal{C})$ and a simple level zero component $\Omega_{[\bar{\chi}]}:=\Omega_{\mathcal{D}_{m}([\bar{\chi}])} \subset \Omega\left(A^{\times}\right)$.

1.2 Proposition. The mapping $\langle\phi\rangle \backslash X\left(k_{n}^{\times}\right) \ni[\bar{\chi}] \mapsto \Omega_{[\bar{\chi}]}=\Omega_{\mathcal{D}_{m}([\bar{\chi}])}$ gives a bijection from $\langle\phi\rangle \backslash X\left(k_{n}^{\times}\right)$to the set of simple level zero connected components of $\Omega\left(A^{\times}\right)$. If $[\bar{\chi}]$ corresponds to the degree $m$ divisor $e^{\prime}[\sigma]$, then $\left(\mathfrak{A}_{e^{\prime}}^{\times}, \sigma^{\otimes e^{\prime}}\right)$ is a type for the component $\Omega_{[\bar{\chi}]}$. If $\Pi \in \operatorname{Irr}\left(\Omega_{[\bar{\chi}]}\right)$ is an irreducible representation with cuspidal support in $\Omega_{[\bar{\chi}]}$, then its central character $\omega_{\Pi}$ is a tame character of $F^{\times}$with $\bar{\omega}_{\Pi}=\left.\bar{\chi}\right|_{k^{\times}} \in X\left(k^{\times}\right)$.

Proof. The proof follows from $[\mathrm{GSZ}](5.4,5.5)$. In particular, the central character $\omega_{\Pi}$ is tame because $\Pi$ is a level zero representation and the reduction $\bar{\omega}_{\Pi}$ is constructed by restricting to the units of $F^{\times}$and factoring mod the principal units. It is obviously determined by the type. From Proposition 1.1 we obtain $\bar{\omega}_{\Pi}=\left.\left(\bar{\chi}_{d f^{\prime}}\right)^{e^{\prime}}\right|_{k^{\times}}=\left.\bar{\chi}\right|_{k^{\times}}$.

\section{$\S 2$. A Parameterization of the Set of Inertial Classes $\mathcal{R}_{0}^{2}\left(A^{\times}\right) / \sim$.}

We shall continue to use a "bar" over a Greek letter to denote a multiplicative character of a finite field; e.g. if $\chi$ is a tame multiplicative character of a p-adic field, then $\bar{\chi}$ usually will denote its "reduction", i.e. the reduction of its restriction to the group of units.

2.1 Proposition. Let $\Omega=\Omega(\mathcal{D})$ be a level zero Bernstein component and assume that $\mathcal{D}$ is not a simple divisor. Then $\mathcal{R}^{2}\left(A^{\times}\right) \cap \operatorname{Irr}(\Omega)=\emptyset$.

Proof. We give two proofs for this Proposition, the first in the context of Bushnell/Kutzko theory and the second based on a theorem of Harish-Chandra. The second proof will allow us to associate to any irreducible discrete series of $A^{\times}$a unitary supercuspidal "base representation".

(i) Let $\Omega \subset \Omega\left(A^{\times}\right)$be a level zero connected component. Assume that $\Omega=\Omega(\mathcal{D})$, where the degree $m$ divisor $\mathcal{D}$ is not simple. By [GSZ], Proposition 5.3 and Theorem 5.4 the types $\left(\mathfrak{A}^{\times}, \tau\right)$ associated to $\Omega$ all have the same divisor $\Delta(\tau)=$ 
$\sum_{[\sigma]} r_{[\sigma]}(\tau)[\sigma]$ with $r_{[\sigma]}>0$ for at least two distinct orbits $[\sigma]$. Then we consider the proper Levi subgroup $\widetilde{M}=\prod_{[\sigma]} G L_{r_{[\sigma]}(\tau) \cdot d(\sigma)}(D) \subset A^{\times}$. Using the Hecke algebra isomorphism [GSZ]1.9 we obtain from [BK2](8.4) that the parabolic induction from $\widetilde{M}$ to $G$ is an equivalence between the categories $\mathcal{M}_{\widetilde{M}}(\Omega)$ and $\mathcal{M}_{G}(\Omega)$, where $\Omega=\Omega(\mathcal{D})$ is interpreted as a connected component of the Bernstein spec$\operatorname{trum} \Omega(\widetilde{M})$ in the obvious way. This implies that $\Pi \in \operatorname{Irr}(\Omega)$ is always parabolically induced from $\tilde{M}$ and therefore $\Pi$ cannot be discrete series. Indeed either by AMT (or, to give a local argument, by Harish-Chandra's Selberg principle) the character of a discrete series representation cannot completely vanish on the regular elliptic set, whereas the character of a parabolically induced representation does vanish for regular elliptic elements.

(ii) For $\mathcal{D}=\sum_{\pi \in \mathcal{C}} \mathrm{m}_{\pi} \pi \in \operatorname{Div}^{+}(\mathcal{C})$ consider the cuspidal pair

$$
\left(M_{\mathcal{D}}=\prod_{\pi \in \mathcal{C}} G L_{d(\pi)}(D)^{\times \mathrm{m}_{\pi}}, \pi_{\mathcal{D}}=\otimes_{\pi \in \mathcal{C}} \pi^{\otimes \mathrm{m}_{\pi}}\right)
$$

and assume that the connected component $\Omega(\mathcal{D})$ is generated by the $A^{\times}$orbit of $\left(M_{\mathcal{D}}, \pi_{\mathcal{D}}\right)$. Assume, moreover, that the supercuspidal support of $\Pi \in \mathcal{R}^{2}\left(A^{\times}\right)$lies in $\Omega(\mathcal{D})$, hence that it is the $A^{\times}$orbit of $\left(M_{\mathcal{D}}, \pi_{0}\right)$, where $\pi_{0}$ is an unramified twist of $\pi_{\mathcal{D}}$. Let $\pi_{0}=\pi_{1} \otimes \psi$ be the decomposition of $\pi_{0}$, where $\pi_{1}$ is unitary supercuspidal and $\psi$ is a positive, real-valued character of $M:=M_{\mathcal{D}}$. Let $T$ be the maximal split torus in the center of $M$ and let $W(T):=\mathcal{N}_{A^{\times}}(M) / M$ be the corresponding Weyl group. Let $W\left(\pi_{1}\right) \subset W(T)$ be the subgroup consisting of elements which fix the class of $\pi_{1}$. Since $\Pi \in \mathcal{R}^{2}\left(A^{\times}\right)$, it follows from [Si], Corollary 5.4.5.8 that $W\left(\pi_{1}\right)$ contains reflections with respect to $r-1$ linearly independent $T$-roots of $A^{\times}$, where $r$ is the number of diagonal blocks of $M\left(r=\sum_{\pi \in \mathcal{C}} \mathrm{m}_{\pi}\right)$. It is easy to see that this implies that $W(T)$ must permute the $r$ blocks of $M$, so $W(T) \cong \mathfrak{S}_{r}$ is the symmetric group on $r$ letters and, moreover, $W\left(\pi_{1}\right)=W(T)$. This implies that $\pi_{1}=\Sigma^{\otimes r}$, where the supercuspidal representation $\Sigma$ of $G L_{m / r}(D)$ does not depend upon the choice of $\pi_{0}$, because $\pi_{0}$ and $\pi_{1}$ are unique up to the action of $W(T)$ and $\Sigma^{\otimes r}$ is $W(T)$-invariant. Since $\Sigma^{\otimes r}=\pi_{1}$ is an unramified twist of $\pi_{\mathcal{D}}$, it follows that $\mathcal{D}=m_{\pi} \pi=r \pi$ for a single $\pi \in \mathcal{C}$. In particular, it follows that $\mathcal{D}$ must be a simple divisor and $\Sigma$ an unramified twist of $\pi$. From (0.3) it follows that, when $\Pi$ is level zero, then $\mathcal{D}=r \pi$, where $\pi=\pi_{[\sigma]}$ for some orbit $[\sigma] \in \overline{\mathcal{C}}\left(k_{D}\right)$.

\section{Remarks.}

(i) The second argument applies to general discrete series representations and proves that a discrete series representation must lie in a Bernstein component corresponding to a simple divisor.

(ii) If $\Pi \in \mathcal{R}^{2}\left(A^{\times}\right)$, we write ${ }^{b} \Pi:=\Sigma$, where $\Sigma$ is as constructed in Proof (2) above. We call ${ }^{b} \Pi$ the supercuspidal base representation of $\Pi$.

2.2 Corollary. If $\mathcal{R}_{0}^{2}\left(A^{\times}\right) \cap \operatorname{Irr}(\Omega) \neq \emptyset$, then $\Omega=\Omega_{[\bar{\chi}]}$ for some Galois orbit $[\bar{\chi}] \in X\left(k_{n}^{\times}\right)$.

Proof. Proposition 2.1 implies that, if $\mathcal{R}_{0}^{2}\left(A^{\times}\right) \cap \Omega \neq \emptyset$, then $\Omega=\Omega(\mathcal{D})$ for some simple divisor $\mathcal{D}$. On the other hand, Proposition 1.2 gives a parameterization of the set of simple level zero components such that each is of the form $\Omega_{\bar{\chi}}$ for some $\bar{\chi} \in X\left(k_{n}^{\times}\right)$. 
2.3 Definition. We write $\mathcal{S}_{\bar{\chi}}^{A} \subset \operatorname{Irr}\left(\Omega_{[\bar{\chi}]}\right)$ for the set of irreducible discrete series representations which have supercuspidal support in $\Omega_{[\bar{\chi}]}$.

From Proposition 2.1, Proof (ii), it follows that the base representation mapping $\Pi \mapsto{ }^{b} \Pi$ is defined for any $\Pi \in \mathcal{R}^{2}\left(A^{\times}\right)$. We want to give some of the properties of this mapping but we shall be able here to give proofs only in the level zero case, to which we now return.

Let $\mathcal{R}_{0}^{0}\left(A^{\times}\right) \subset \mathcal{R}_{0}^{2}\left(A^{\times}\right)$denote the subset consisting of unitary supercuspidal level zero representations.

2.4 Proposition. The base representation map defines a bijection

$$
\mathcal{R}_{0}^{2}\left(A^{\times}\right) \ni \Pi \longmapsto{ }^{b} \Pi \in \bigsqcup_{s \mid m} \mathcal{R}_{0}^{0}\left(G L_{s}(D)\right) .
$$

Proof. Injectivity: Let $\Sigma:={ }^{b} \Pi_{1}={ }^{b} \Pi_{2} \in \mathcal{R}_{0}^{0}\left(G L_{s}(D)\right)$, let $r=m / s$, and let $M=\left[G L_{s}(D)\right]^{r}$ be a block diagonal Levi subgroup of $A^{\times}$. Since the supercuspidal supports of $\Pi_{1}$ and $\Pi_{2}$ are inertially equivalent to $\Sigma^{\otimes r}$, it follows that $\Pi_{1}, \Pi_{2} \in$ $\operatorname{Irr}(\Omega)$ for the same connected component $\Omega$ of the Bernstein spectrum. From the classification of these components given in Proposition 1.2, we know that $\Omega=\Omega_{[\bar{\chi}]}$, i.e. $\Pi_{1}, \Pi_{2} \in \mathcal{S}_{\bar{\chi}}^{A}$ for some $[\bar{\chi}] \in X\left(k_{n}^{\times}\right) /\langle\phi\rangle$. Applying Proposition 2.8, we see that $\Pi_{1}=\Pi_{2} \tilde{\eta}$ for some unramified character $\tilde{\eta}=\eta \circ \operatorname{Nrd}_{A \mid F}$ of $A^{\times}$. We also write $\tilde{\eta}^{\prime}=\eta \circ \operatorname{Nrd}_{A^{\prime} \mid F}$ for $A^{\prime}=M_{s}(D)$. Let $P=M \ltimes U$ be the standard (upper triangular) parabolic subgroup of $A^{\times}$with $M$ as Levi factor. Then $\Pi_{2} \subset i_{A^{\times}, P}\left(\Sigma^{\otimes r} \psi\right)$, where $\psi$ is a positive, real-valued unramified character of $P / U=M$ and $i_{A \times, P}$ denotes normalized (unitary) parabolic induction. Therefore,

$$
\Pi_{1}=\Pi_{2} \otimes \tilde{\eta} \subset \tilde{\eta} \otimes i_{A^{\times}, P}\left(\Sigma^{\otimes r} \psi\right)=i_{A^{\times}, P}\left(\left(\tilde{\eta}^{\prime} \Sigma\right)^{\otimes r} \psi\right),
$$

which implies that ${ }^{b} \Pi_{1}=\tilde{\eta}^{\prime} \Sigma$. Our hypothesis further implies that $\Sigma=\tilde{\eta}^{\prime} \Sigma$, i.e. that $\Pi_{1}$ and $\Pi_{2}$ have the same supercuspidal support. We may conclude from this that $\Pi_{1}=\Pi_{2}$, since it is known that the composition series of $i_{A^{\times}, P}\left(\Sigma^{\otimes r} \psi\right)$ contains only one discrete series component.

Surjectivity: Assume that $r s=m$ and that $\pi \in \mathcal{R}_{0}^{0}\left(G L_{s}(D)\right)$. From [GSZ], Proposition 5.5(ii) we know that $\pi$ is an unramified twist of $\pi_{[\sigma]}$ for some $[\sigma] \in$ $G L_{s}\left(k_{D}\right)_{\text {cusp }}^{\wedge} /\langle\phi\rangle$. By Proposition 1.1 there is a unique $[\bar{\chi}] \in\langle\phi\rangle \backslash X\left(k_{n}^{\times}\right)$such that $\mathcal{G}_{m}([\bar{\chi}])=[\sigma]$. This means that $\mathcal{D}_{m}([\bar{\chi}])=r \pi_{[\sigma]}$. Again applying Proposition 2.8 we see that $\mathcal{S}_{\bar{\chi}}^{A} \neq \emptyset$. The supercuspidal support of $\Pi \in \mathcal{S}_{\bar{\chi}}^{A}$ is an unramified twist of $\pi_{[\sigma]}^{\otimes r}$ and ${ }^{b} \Pi$ is an unramified twist of $\pi_{[\sigma]}$. But for an unramified character $\tilde{\eta}$ as above we have seen that ${ }^{b}(\Pi \otimes \tilde{\eta})={ }^{b} \Pi \otimes \tilde{\eta}^{\prime}$. Thus we can realize any unramified unitary twist of $\pi_{[\sigma]}$, in particular $\pi$, as the base representation of some $\Pi \in \mathcal{R}_{0}^{2}\left(A^{\times}\right)$.

Remark. Let $\Omega \subset \Omega\left(A^{\times}\right)$be a connected component of the Bernstein spectrum. The proof of Proposition 2.4 depended upon the following two basic facts:

(i) The set $\operatorname{Irr}(\Omega)$ contains at most one unramified twist class of discrete series representations. We used this fact to prove injectivity.

(ii) There exists an unramified twist class of discrete series representations in $\operatorname{Irr}(\Omega)$ when $\Omega=\Omega(\mathcal{D})$ for a simple divisor $\mathcal{D}$. We needed this fact to prove surjectivity. 
We proved our assertion only for level zero representations and depended upon Proposition 2.8 for the two facts. We remark here that both facts are known in complete generality. In particular, they follow from AMT ([DKV], Theorem B.2.b), which reduces these assertions to the split case.

Applying (0.3), which defines a bijection of $\bigsqcup_{s \mid m} G L_{s}\left(k_{d}\right)_{\text {cusp }}^{\wedge}$ to the set of inertial classes $\bigsqcup_{s \mid m} \mathcal{R}_{0}^{0}\left(G L_{s}(D)\right) / \sim$, we may then use (1.1) and Proposition 2.4 to parameterize the set of level zero inertial classes $\mathcal{R}_{0}^{2}\left(A^{\times}\right) / \sim$ by the set of Galois orbits $X\left(k_{n}^{\times}\right) /\langle\phi\rangle$. We thus obtain a map $\bar{\chi} \mapsto \mathcal{S}_{\bar{\chi}}^{A}$ (See $\left.(2.5)\right)$.

In the following we use notation explained in the paragraph prior to Proposition 1.2 .

2.5 Proposition. Assume that $\mathcal{D}_{m}([\bar{\chi}])=e^{\prime} \pi_{[\sigma]}$, i.e. $\Omega_{[\bar{\chi}]} \subset \Omega\left(A^{\times}\right)$is the connected component which contains the cuspidal pair $\left(M, \pi_{[\sigma]}^{\otimes e^{\prime}}\right)$ with respect to the Levi subgroup $M=\left[G L_{f^{\prime}}(D)\right]^{\times e^{\prime}} \subset A^{\times}$. For any $\Pi \in \mathcal{S}_{\bar{\chi}}^{A}$ the normalized Jacquet functor $r_{M, A^{\times}}(\Pi)$ is irreducible; specifically, $r_{M, A^{\times}}(\Pi)=\pi_{[\sigma]}^{\otimes e^{\prime}} \cdot \psi$ for some unramified character $\psi$ of $M$ which is not unitary.

Proof. The type $\left(\mathfrak{A}_{e^{\prime}}^{\times}, \tau\right)$ for $\Omega_{[\bar{\chi}]}$ is a cover of $\left(M \cap \mathfrak{A}_{e^{\prime}}^{\times}, \sigma^{\otimes e^{\prime}}\right)$, which is a type for the supercuspidal component $\Omega_{[\bar{\chi}]}(M) \subset \Omega(M)$ (see [GSZ] 5.3 and its proof). Therefore, by [BK2](7.9)(ii), we have a natural isomorphism of complex vector spaces

$$
\operatorname{Hom}_{\mathfrak{A}_{e^{\prime}}^{\times}}(\tau, \Pi) \cong \operatorname{Hom}_{\mathfrak{A}_{e^{\prime}}^{\times} \cap M}\left(\tau, r_{M, A} \times(\Pi)\right) .
$$

Let $\omega$ denote the central character of $\Pi$. Then we also have the natural isomorphism

$$
\operatorname{Hom}_{\mathfrak{A}_{e^{\prime}}^{\times}\left\langle\varpi_{F}\right\rangle}\left(\tau_{\omega}, \Pi\right) \cong \operatorname{Hom}_{\mathfrak{A}_{e^{\prime}}^{\times}}(\tau, \Pi) .
$$

Since $\Pi$ is irreducible, $\operatorname{Hom}_{\mathfrak{A}_{e^{\prime}}^{\times}\left\langle\varpi_{F}\right\rangle}\left(\tau_{\omega}, \Pi\right)$ is a finite-dimensional simple module over the Hecke algebra $\mathcal{H}\left(A^{\times}, \mathfrak{A}_{e^{\prime}}^{\times}\left\langle\varpi_{F}\right\rangle, \tau_{\omega}\right)$. Since $\Pi$ is a discrete series representation, we may apply Proposition A9 with $(K, \rho)=\left(\mathfrak{A}_{e^{\prime}}^{\times}\left\langle\varpi_{F}\right\rangle, \tau_{\omega}\right)$. This implies that the modules in (2.2) are complex vector spaces of dimension one.

Considering now the right side of (2.1), we see that $r_{M, A^{\times}}(\Pi)$ contains the type $\left(\mathfrak{A}_{e^{\prime}}^{\times}, \tau\right)$ with multiplicity one and therefore $r_{M, A^{\times}}(\Pi)$ contains an irreducible component $\pi_{[\sigma]}^{\otimes e^{\prime}} \cdot \psi$ and indeed only one. The character $\psi$ cannot be unitary, since if it were unitary, then $\Pi$ would be a subrepresentation of a unitarily induced representation and therefore not a discrete series representation. Moreover, any other component of $r_{M, A} \times(\Pi)$ has to be supercuspidal and conjugate to $\pi_{[\sigma]}^{\otimes e^{\prime}} \cdot \psi$ under the normalizer of $M$, i.e. it has to be of the form $\pi_{[\sigma]}^{\otimes e^{\prime}} \cdot \psi^{\prime}$ (see e.g. [BR], III.2 Theorem 18). Any such component contains $\left.\tau\right|_{M \cap \mathfrak{A}_{e^{\prime}}^{\times}}$. By [BK2](7.9) this is impossible since $\tau$ occurs as a component of $\left.\Pi\right|_{\mathfrak{A}_{e^{\prime}}^{\times}}$only once. Thus $r_{M, A^{\times}}(\Pi)=\pi_{[\sigma]}^{\otimes e^{\prime}} \cdot \psi$ and is irreducible.

Remark. Since $r_{M, A^{\times}}(\Pi)$ is irreducible, it belongs to the supercuspidal support of $\Pi$. Therefore, we have

$$
r_{M, A \times}(\Pi)={ }^{b} \Pi^{\otimes e^{\prime}} \psi_{1}=\left(\pi_{[\sigma]} \tilde{\eta}^{\prime}\right)^{\otimes e^{\prime}} \psi_{1},
$$


where $\psi_{1}$ is a positive, real-valued character and $\tilde{\eta}^{\prime}$ is a unitary unramified character.

As before, $\left(\mathfrak{A}_{e^{\prime}}^{\times}, \tau\right)$ is the type such that $\tau=\sigma^{\otimes e^{\prime}}$. We write

$$
\tau^{\nu_{1}, \ldots, \nu_{e^{\prime}}}:=\sigma^{\phi^{\nu_{1}}} \otimes \cdots \otimes \sigma^{\phi^{\nu} e^{\prime}} .
$$

The set of tuples $\left(\nu_{1}, \ldots, \nu_{e^{\prime}}\right) \in(\mathbb{Z} /((d, f)))^{e^{\prime}}$ parameterizes the $(d, f)^{e^{\prime}}$ conjugates of $\tau$.

2.6 Corollary. Let $(\Pi, V) \in \mathcal{S}_{\bar{\chi}}^{A}$ and let $V^{1+\mathfrak{P}_{e^{\prime}}}$ denote the subspace of $V$ consisting of all $\left(1+\mathfrak{P}_{e^{\prime}}\right)$-fixed vectors, where $\mathfrak{P}_{e^{\prime}}$ denotes the Jacobson radical of $\mathfrak{A}_{e^{\prime}}$. As a representation of $\overline{\mathfrak{A}}_{e^{\prime}}^{\times}$the space $V^{1+\mathfrak{P}_{e^{\prime}}}$ decomposes as $\oplus \tau^{\nu_{1}, \ldots, \nu_{e^{\prime}}}$, where, for $0 \leq \nu_{1}, \ldots, \nu_{e^{\prime}}<(d, f)$, each representation occurs with multiplicity one.

Proof. From [GSZ](5.5)(i) we know that each of the above types occurs and with the same multiplicity. As we have seen in the proof of $2.4, \tau$ occurs simply in $\left.\Pi\right|_{\mathfrak{A}_{e^{\prime}}^{\times}}$. Therefore, the same is true for each of the types $\tau^{\nu_{1}, \ldots, \nu_{e}^{\prime}}$.

We consider the unramified extension $F_{n} \mid F$ of degree $n$ and we write $X_{t}\left(F_{n}^{\times}\right)$ for the set of tamely ramified characters of $F_{n}^{\times}$. Then we have a reduction map

$$
X_{t}\left(F_{n}^{\times}\right) \ni \chi \longmapsto \bar{\chi} \in X\left(k_{n}^{\times}\right),
$$

where $\bar{\chi}$ denotes $\left.\chi\right|_{o_{n}^{\times}}$regarded as a character of $k_{n}^{\times}$. Here we identify $k_{n}$ with the residual field of $F_{n}$ and we use the assumption that $\chi$ is a tame character. Under these assumptions the $\bar{\chi}$ is well defined; we call $\bar{\chi}$ the reduction of $\chi$. Noting that $F_{n}$ has a prime element in $F$, we see that (2.3) is compatible with the Galois action of $\operatorname{Gal}\left(F_{n} \mid F\right) \cong \operatorname{Gal}\left(k_{n} \mid k\right)=\langle\phi\rangle$, the lengths of orbits being preserved. Moreover, the character $\chi \in X_{t}\left(F_{n}^{\times}\right)$is uniquely determined by its reduction $\bar{\chi} \in X\left(k_{n}^{\times}\right)$and its restriction $\chi_{F} \in X_{t}\left(F^{\times}\right)$. For $\Pi \in \mathcal{S}_{\bar{\chi}}^{A}$ it follows from Definition 2.3 and from the last statement of Proposition 1.2 that the central character $\omega_{\Pi} \in X_{t}\left(F^{\times}\right)$has the reduction $\bar{\omega}_{\Pi}=\left.\bar{\chi}\right|_{k^{\times}}$. From this we obtain a partition $\mathcal{S}_{\bar{\chi}}^{A}=\cup_{\chi} \mathcal{S}_{\chi}^{A}$, where $\chi \in X_{t}\left(F_{n}^{\times}\right)$runs over all tame characters which have the prescribed reduction $\bar{\chi}$ and where:

2.7 Definition. For $\chi \in X_{t}\left(F_{n}^{\times}\right)$set $\mathcal{S}_{\chi}^{A}:=\left\{\Pi \in \mathcal{S}_{\bar{\chi}}^{A}: \omega_{\Pi}=\chi_{F}\right\}$.

Like $\mathcal{S}_{\bar{\chi}}^{A}$ (cf. Def. 2.3) the definition of $\mathcal{S}_{\chi}^{A}$ depends only upon the $\langle\phi\rangle$-orbit of $\chi$.

2.8 Proposition. If $\chi \in X_{t}\left(F_{n}^{\times}\right)$generates $a\langle\phi\rangle$-orbit $[\chi]$ of length $f$, then $\mathcal{S}_{\chi}^{A}$ is a set of order $e=n / f$. In particular, the group of unramified characters of order dividing $n$ acts transitively on $\mathcal{S}_{\chi}^{A}$. The set $\mathcal{S}_{\bar{\chi}}^{A}$ is a single unramified twist class.

Proof. We note that $\mathcal{S}_{\chi}^{A}$ consists of all $\Pi \in \mathcal{S}_{\bar{\chi}}^{A}$ which have a fixed central character. By Proposition 1.2 the representations of $\mathcal{S}_{\chi}^{A}$ admit the type $\left(\mathfrak{A}_{e^{\prime}}^{\times}, \tau\right)$, where $\tau=$ $\sigma^{\otimes e^{\prime}}$. Therefore, we may apply Corollary A10 with $r=e^{\prime}$ and $l=(d, f)$, which according to Proposition 1.1 is $|[\sigma]|$. This implies that $d r / l=d e^{\prime} /(d, f)=e$, in which the second equality follows from $e f=d m$ and $e^{\prime}=(e, m)$. If $\eta \in X\left(F^{\times}\right)$and $\tilde{\eta}=\eta \circ \operatorname{Nrd}_{A \mid F}$, then we know that $\omega_{\Pi \otimes \tilde{\eta}}=\omega_{\Pi} \otimes \eta^{n}$, which implies that the group of unramified characters $\eta$ of order dividing $n$ acts on each set $\mathcal{S}_{\chi}^{A}$. Therefore, it suffices to show that, for $\Pi \in \mathcal{S}_{\bar{\chi}}^{A}$, we have $\Pi \otimes \tilde{\chi}_{F}=\Pi$ if and only if $\chi_{F}^{f}=1$. In this 
case, since $n / f=e$, we see that all $e$ inequivalent discrete series representations with the same central character lie in a single unramified twist class. Since the group of unramified characters of $A^{\times}$acts transitively on the set of all $\mathcal{S}_{\chi}$ for which the reduction of $\chi$ is $\bar{\chi}$, it follows that $\mathcal{S}_{\bar{\chi}}^{A}$ is a single unramified twist class.

Let us proceed with the proof that $\Pi \otimes \tilde{\eta}=\Pi$ if and only if $\eta^{f}=1$. From Proposition 2.4 and the comments immediately prior to Proposition 1.2, which explain how to construct a degree $m$ simple divisor from a cuspidal divisor $e^{\prime}[\sigma]$, where $e^{\prime} d(\sigma)=e^{\prime} f^{\prime}=m$, it follows that the supercuspidal support of $\Pi \in \mathcal{S}_{\bar{\chi}}^{A}$ has the form $\pi_{[\sigma]}^{\otimes e^{\prime}} \cdot \psi$, where $\psi$ is some non-unitary unramified character of $M=$ $\left(G L_{f^{\prime}}(D)\right)^{\times e^{\prime}}$. By Jacquet's subrepresentation theorem $\Pi \subset \operatorname{Ind}_{P}^{A^{\times}}\left(\pi_{[\sigma]}^{\otimes e^{\prime}} \cdot \psi\right)$, where $P=M \ltimes U_{P}$ is a parabolic subgroup of $A^{\times}$and the induction is normalized. Thus, $\Pi \cdot \tilde{\eta}$ is contained in $\operatorname{Ind}_{P}^{A^{\times}}\left(\left.\pi_{[\sigma]}^{\otimes e^{\prime}} \cdot \psi \cdot \tilde{\eta}\right|_{M}\right)$. From the irreducibility of the Jacquet module we see that $\Pi \otimes \tilde{\eta}=\Pi$ implies that

$$
\pi_{[\sigma]}^{\otimes e^{\prime}}=\left.\pi_{[\sigma]}^{\otimes e^{\prime}} \tilde{\eta}\right|_{M}
$$

Furthermore $\left.\operatorname{Nrd}_{A \mid F}\right|_{M}=\left(\operatorname{Nrd}_{f^{\prime}}\right)^{\otimes e^{\prime}}$, where $\operatorname{Nrd}_{f^{\prime}}$ denotes the reduced norm mapping $M_{f^{\prime}}(D) \mid F$. Therefore from (2.4) we obtain

$$
\pi_{[\sigma]}=\pi_{[\sigma]} \cdot\left(\eta \circ \mathrm{Nrd}_{f^{\prime}}\right)
$$

for the supercuspidal representation $\pi_{[\sigma]}$ of $G L_{f^{\prime}}(D)$. With this justification we reduce to the supercuspidal case $\Pi=\pi_{[\sigma]}, f^{\prime}=m$, and $e^{\prime}=1$. In this case from [GSZ]5.1 we know that $\Pi=\operatorname{cInd}(\tilde{\sigma})$, where $\tilde{\sigma}$ is an extension of $\sigma$ to $\left\langle\varpi^{l}\right\rangle \mathfrak{A}_{1}^{\times}$. Here and in $[\mathrm{GSZ}] l=|[\sigma]|$. Thus $l=(d, f)$, since $\sigma$ corresponds to $[\bar{\chi}]$ of length $f$ (see Proposition 1.1). The reduced norm Nrd induces an injection

$$
\operatorname{Nrd}_{A \mid F}:\left\langle\varpi^{l}\right\rangle \mathfrak{A}_{1}^{\times} / \mathfrak{A}_{1}^{\times} \hookrightarrow F^{\times} / \mathfrak{o}_{F}^{\times}
$$

with image of index $m l=f^{\prime}(d, f)=f$. Therefore,

$$
\tilde{\eta} \otimes \operatorname{cInd}(\tilde{\sigma})=\operatorname{cInd}(\tilde{\eta} \otimes \tilde{\sigma})=\operatorname{cInd}(\tilde{\sigma})
$$

which is true if and only if $\tilde{\eta} \otimes \tilde{\sigma}=\tilde{\sigma}$, if and only if $\eta^{f}=1$.

Writing $\mathcal{R}_{0}^{2}\left(A^{\times}\right) / \sim$ for the set of inertial equivalence classes of level zero discrete series representations, we now have the bijection

$$
\langle\phi\rangle \backslash X\left(k_{n}^{\times}\right) \ni \bar{\chi} \longmapsto \mathcal{S}_{\bar{\chi}}^{A} \in \mathcal{R}_{0}^{2}\left(A^{\times}\right) / \sim
$$

In concluding this section, we lift (2.5) to a bijection

$$
\operatorname{Gal}\left(F_{n} \mid F\right) \backslash X_{t}\left(F_{n}^{\times}\right) \ni[\chi] \longmapsto \mathcal{S}_{\chi}^{A} \in \mathcal{R}_{0}^{2}\left(A^{\times}\right) / \approx,
$$

where we set $\Pi_{1} \approx \Pi_{2}$ if $\Pi_{1}=\Pi_{2} \otimes \eta$ for some unramified character $\eta$ of $A^{\times}$which is trivial on the center of $A^{\times}$. To formulate our result, which summarizes what has been proved to this point, we also extend $\left(\mathfrak{A}_{e^{\prime}}^{\times}, \tau\right)$ to $\left(\mathfrak{A}_{e^{\prime}}^{\times} F^{\times}, \tau_{\chi_{F}}\right), \tau_{\chi_{F}}$ being the extension of $\tau=\sigma^{\otimes e^{\prime}}$ to $\mathfrak{A}_{e^{\prime}}^{\times}\left\langle\varpi_{F}\right\rangle=\mathfrak{A}_{e^{\prime}}^{\times} F^{\times}$such that $\chi_{F} 1_{\tau}$ is the restriction of $\tau_{\chi_{F}}$ to $F^{\times}$. 
2.9 Theorem. Let $\chi \in X_{t}\left(F_{n}^{\times}\right)$be a unitary character generating the $\operatorname{Gal}\left(F_{n} \mid F\right)$ orbit $[\chi]$ of length $f$ and set $\chi_{F}:=\left.\chi\right|_{F^{\times}}$.

(i) There are precisely $e=n / f$ discrete series representations $\Pi_{1}, \ldots, \Pi_{e}$ of $A^{\times}$with central character $\omega_{\Pi_{i}}=\chi_{F}$ such that $\Pi_{i} \in \mathcal{S}_{\bar{\chi}}^{A}$, i.e. such that $\left.\Pi_{i}\right|_{\mathfrak{A}_{e^{\prime}}^{\times} F^{\times}} \supset \tau_{\chi_{F}}$. This set of representations comprises an equivalence class $\mathcal{S}_{\chi}^{A} \in \mathcal{R}_{0}^{2}\left(A^{\times}\right) / \approx$. The finite set $\mathcal{S}_{\chi}^{A}$ consists of the set of all elements in the inertial class $\mathcal{S}_{\bar{\chi}}^{A}$ with the central character $\chi_{F}$.

(ii) The representations $\Pi_{i} \in \mathcal{S}_{\chi}^{A}$ have supercuspidal support in $\Omega_{[\bar{\chi}]}$. In particular, these representations are supercuspidal if and only if $e^{\prime}=1$.

(iii) The map $\chi \mapsto \mathcal{S}_{\chi}^{A}$ induces a bijection $\operatorname{Gal}\left(k_{n} \mid k\right) \backslash X_{t}\left(F_{n}^{\times}\right) \leftrightarrow \mathcal{R}_{0}^{2}\left(A^{\times}\right) / \approx$ which lifts the bijection (2.5).

Remark. In the case $A=D_{n}$ the injection

$$
F_{n}^{\times} /\left(1+\mathfrak{p}_{F_{n}}\right) \stackrel{\sim}{\longrightarrow} \mathfrak{o}_{D_{n}}^{\times} F^{\times} /\left(1+\mathfrak{p}_{D_{n}}\right) \hookrightarrow D_{n}^{\times} /\left(1+\mathfrak{p}_{D_{n}}\right)
$$

gives $\tau_{\chi}=\chi \in X_{t}\left(F_{n}^{\times}\right)$as a character of $\mathfrak{o}_{D_{n}}^{\times} F^{\times} /\left(1+\mathfrak{p}_{D_{n}}\right)$. Thus we may regard $\mathcal{S}_{\chi}^{D_{n}}$ as the set of irreducible components of the representation $\operatorname{Ind}_{\mathfrak{o}_{D_{n}}^{\times} F^{\times}}^{D_{x}^{\times}}(\chi)$.

\section{$\S$. The Characters $\mathcal{R}_{0}^{2}\left(A^{\times}\right)$at Very Regular Elements.}

Let $(\Pi, V) \in \mathcal{R}_{0}^{2}\left(A^{\times}\right)$and assume that the supercuspidal support of $\Pi$ lies in $\Omega_{[\bar{\chi}]}$. Let $\left(\mathfrak{A}_{e^{\prime}}^{\times}, \tau\right)$, where $\tau=\sigma^{\otimes e^{\prime}}$, be the corresponding type.

We have previously considered the $\overline{\mathfrak{A}}_{e^{\prime}}^{\times}$representation on $V^{1+\mathfrak{P}_{e^{\prime}}}$ (Corollary 2.6). Now we want to study the $\overline{\mathfrak{A}}_{1}^{\times}$representation on $V^{1+\mathfrak{P}_{1}}$.

\subsection{Lemma.}

(i) The Jacquet restriction mapping $r_{\overline{\mathfrak{A}}_{e^{\prime}}^{\times}, \overline{\mathfrak{A}}_{1}^{\times}}: \mathcal{M}\left(\overline{\mathfrak{A}}_{1}^{\times}\right) \rightarrow \mathcal{M}\left(\overline{\mathfrak{A}}_{e^{\prime}}^{\times}\right)$induces a bijection between the set of irreducible constituents of $V^{1+\mathfrak{P}_{1}}$ and the set

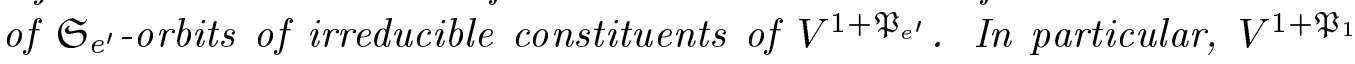
decomposes simply.

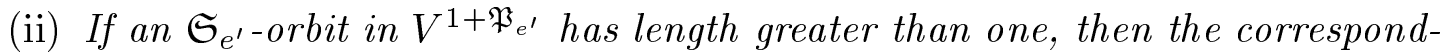
ing constituent of $V^{1+\mathfrak{P}_{1}}$ is induced from a proper parabolic subgroup of $\overline{\mathfrak{A}}_{1}^{\times}$ and its character vanishes on the regular elliptic set of $\overline{\mathfrak{A}}_{1}^{\times}$.

(iii) If an orbit has length equal one, then the corresponding constituent of $V^{1+\mathfrak{P}_{1}}$ is a generalized Steinberg representation with cuspidal support $\left(\sigma^{\phi^{\nu}}\right)^{\otimes e^{\prime}}$.

Proof. (i) Since $\left(\mathfrak{A}_{e^{\prime}}^{\times}, \tau\right)$ is a type in $(\Pi, V)$ and any cuspidal level zero type of $(\Pi, V)$ corresponds, up to conjugacy, to a representation of $\overline{\mathfrak{A}}_{e^{\prime}}^{\times}$, the mapping $r:=r_{\overline{\mathfrak{A}}_{e^{\prime}}^{\times}, \overline{\mathfrak{A}}_{1}^{\times}}$ does not annihilate any irreducible $\overline{\mathfrak{A}}_{1}^{\times}$-submodule of $V^{1+\mathfrak{P}_{1}}$. Obviously, we may regard $V^{1+\mathfrak{P}_{e^{\prime}}}=r\left(V^{1+\mathfrak{P}_{1}}\right)$. Therefore, since, by Corollary 2.6, the $\overline{\mathfrak{A}}_{e^{\prime}}^{\times}$-module $V^{1+\mathfrak{P}_{e^{\prime}}}$ decomposes simply, it follows that the $\overline{\mathfrak{A}}_{1}^{\times}$-module $V^{1+\mathfrak{P}_{1}}$ decomposes simply too. Let $\pi$ be an irreducible $\overline{\mathfrak{A}}_{1}^{\times}$representation occurring in $V^{1+\mathfrak{P}_{1}}$. Every constituent of $r(\pi)$ occurs with multiplicity one and if $\pi^{\prime}$ is any other constituent of $V^{1+\mathfrak{P}_{1}}$, then $r(\pi)$ and $r\left(\pi^{\prime}\right)$ are disjoint. By Frobenius reciprocity a constituent $\tau^{\prime}$ of $V^{1+\mathfrak{P}_{e^{\prime}}}$ occurs in $r(\pi)$ if and only if $\pi \subset \operatorname{Ind}_{\mathfrak{A}_{e^{\prime}}^{\times}}^{\mathfrak{A}_{1}^{\times}} \tau^{\prime}$. For $i=1,2$ let $\tau_{i}=\sigma_{1}^{i} \otimes \cdots \otimes \sigma_{e^{\prime}}^{i}$ be a constituent of $V^{1+\mathfrak{P}_{e^{\prime}}}$. Let $T_{i}:=\left\{\sigma_{1}^{i}, \ldots, \sigma_{e^{\prime}}^{i}\right\}$ be the corresponding multiset, 
$i=1,2$. If $T_{1} \neq T_{2}$ (i.e. the two representations $\tau_{1}$ and $\tau_{2}$ are not just rearrangements of the same tensor factors), then, as is well known, it follows from the Bruhat decomposition of the group $\overline{\mathfrak{A}}_{1}^{\times}$, the two induced representations Ind $\frac{\overline{\mathfrak{A}}_{1}^{\times}}{\overline{\mathfrak{A}}_{e^{\prime}}^{\times}} \tau_{i}$ are disjoint. Therefore the set of constituents of $r(\pi)$ lies in a single $\mathfrak{S}_{e^{\prime} \text {-orbit, i.e. }}$ $\pi$ has associated to it by $r$ a single multiset $T$. On the other hand, assume that $T_{1}=T_{2}$ and assume that $\sigma_{k}^{1}=\sigma_{k}^{2}$ for all $k \neq j, j+1$ and that $\sigma_{j}^{i}=\sigma_{j+1}^{i^{\prime}}$ for $i \neq i^{\prime}$ and $1 \leq i, i^{\prime} \leq 2$. Let $\mathfrak{A}^{j} \subset M_{e^{\prime}}\left(M_{f^{\prime}}\left(O_{D}\right)\right)$ be the standard hereditary order which differs from $\mathfrak{A}_{e^{\prime}}$ only in that the block $j+1, j$ is allowed to consist of arbitrary matrices in $M_{f^{\prime}}\left(O_{D}\right)$. Then

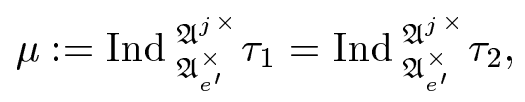

$\mu$ being irreducible. It follows, by transitivity of induction, that

$$
\operatorname{Ind} \underset{\mathfrak{A}_{e^{\prime}}^{\times}}{\mathfrak{A}_{1}^{\times}} \tau_{1}=\operatorname{Ind} \underset{\mathfrak{A}_{e^{\prime}}^{\times}}{\mathfrak{A}_{1}^{\times}} \tau_{2}
$$

and, if $\pi \subset \operatorname{Ind}_{\mathfrak{A}_{e^{\prime}}^{\times}}^{\mathfrak{A}_{1}^{\times}} \tau_{i}$, then $\mu \subset r_{\mathfrak{A}^{j} \times, \overline{\mathfrak{A}}_{1}^{\times}}(\pi)$. Since $r_{\overline{\mathfrak{A}}_{e^{\prime}}^{\times}, \overline{\mathfrak{A}}^{j} \times}(\mu)=\tau_{1}+\tau_{2}$, it follows that $r(\pi)$ contains $\tau_{1}$ if and only if it contains $\tau_{2}$. Since the set of transpositions of the form $j \leftrightarrow j+1\left(1 \leq j<e^{\prime}\right)$ generate $\mathfrak{S}_{e^{\prime}}$, it follows that the set of constituents $r(\pi)$ consists of one full $\mathfrak{S}_{e^{\prime}}$ orbit of constituents of $V^{1+\mathfrak{P}_{e^{\prime}}}$.

(ii) First, if a representation of $\overline{\mathfrak{A}}_{1}^{\times}=G L_{m}\left(k_{d}\right)$ is induced from a proper parabolic subgroup of $G L_{m}\left(k_{d}\right)$, then certainly its character vanishes on the regular elliptic set of $G L_{m}\left(k_{d}\right)$. To prove all of (ii) it is enough to check that the intertwining number of the induced representation $\operatorname{Ind}_{\mathfrak{A}_{e^{\prime}}^{\times}}^{\mathfrak{A}_{1}^{\times}} \sigma_{1}^{\otimes r_{1}} \otimes \cdots \otimes \sigma_{\ell}^{\otimes r_{\ell}}$, where $\sigma_{1}, \ldots, \sigma_{\ell}$ are inequivalent cuspidal representations of $G L_{f^{\prime}}\left(k_{d}\right)$ and $r_{1}+\cdots+r_{\ell}=e^{\prime}$, is equal to the product $\prod_{j=1}^{\ell}\left|\mathfrak{S}_{r_{j}}\right|$. We omit this argument (see e.g. [DM], p. 58, 6.5), which implies that the correct intertwining number is already obtained after inducing to $\prod_{j=1}^{\ell} G L_{r_{j} f^{\prime}}\left(k_{d}\right)$; by transitivity of induction each component of the representation of $\prod_{j=1}^{\ell} G L_{r_{j} f^{\prime}}\left(k_{d}\right)$ induces an irreducible representation of $G L_{m}\left(k_{d}\right)$, since further induction does not produce more intertwining.

(iii) Without loss of generality we may assume that $r(\pi)=\tau$. In this case,

$$
\operatorname{Hom}_{\mathfrak{A}_{e^{\prime}}^{\times}}(\tau, \pi) \subseteq \operatorname{Hom}_{\mathfrak{A}_{e^{\prime}}^{\times}}(\tau, \Pi) .
$$

Since $\Pi \in \mathcal{R}_{0}^{2}\left(A^{\times}\right)$, it follows from Proposition A9 that the right side of this inclusion has complex dimension one. This, together with the fact that the inclusion is non-zero, implies that the inclusion is an isomorphism of one-dimensional complex vector spaces. Now let $\mathcal{H}=\mathcal{H}\left(A^{\times}, \mathfrak{A}_{e^{\prime}}^{\times}\left\langle\varpi_{F}\right\rangle, \tau_{\omega}\right)\left(\omega=\omega_{\Pi}\right), \mathcal{H}^{2}$ the completion of $\mathcal{H}$ (see (a.11) and subsequent remarks), $\mathcal{H}_{0}=\mathcal{H}\left(\mathfrak{A}_{1}^{\times}\left\langle\varpi_{F}\right\rangle, \mathfrak{A}_{e^{\prime}}^{\times}\left\langle\varpi_{F}\right\rangle, \tau_{\omega}\right)$, and $\overline{\mathcal{H}}_{0}$ the reduction of $\mathcal{H}_{0}$ modulo $\left(1+\mathfrak{P}_{1}\right)\left\langle\varpi_{F}\right\rangle$. Since $\Pi$ is discrete series, we see from Proposition A7 that the $\mathcal{H}^{\text {opp }}$-module $\operatorname{Hom}_{\mathfrak{A}_{e^{\prime}}^{\times}}(\tau, \Pi)$ can be embedded into $\mathcal{H}^{2}$. The module structure on $H=\operatorname{Hom}_{\mathfrak{A}_{e^{\prime}}^{\times}}(\tau, \Pi)$ carries over to a convolution action on a subspace of $\mathcal{H}^{2}$. In the unramified split case this embedding sends $H$ to the space $\mathbb{C} \cdot e_{S t}$, where $e_{S t}$ denotes the $\mathcal{I}$ bi-invariant matrix coefficient of a 
Steinberg representation. Via our isometric isomorphisms we bring this function back to a function, also to be denoted $e_{S t}$, for the general case. For $\varphi \in \mathcal{H}$ we have $\varphi * e_{S t}=\lambda(\varphi) e_{S t}$, where $\lambda: \mathcal{H} \rightarrow \mathbb{C}$ is the character of $\mathcal{H}$ associated to our one-dimensional module. By restriction we obtain a character $\lambda_{0}$ of $\mathcal{H}_{0}$. Since convolution by $\varphi \in \mathcal{H}_{0}$ commutes with restriction to $\mathfrak{A}_{1}^{\times}\left\langle\varpi_{F}\right\rangle$, this character can also be represented by $\varphi * e_{S t, 0}=\lambda_{0}(\varphi) e_{S t, 0}$, where $e_{S t, 0}$ denotes the restriction of $e_{S t}$ to $\mathfrak{A}_{1}^{\times}\left\langle\varpi_{F}\right\rangle$. Now we can pass to the reduction $\overline{\mathcal{H}}_{0}$ and observe that $\bar{\varphi} * e_{s t}=\bar{\lambda}_{0}(\bar{\varphi}) e_{s t}$, where $e_{s t}$ denotes the reduction of $e_{S t, 0}$. Since $e_{s t}$ is the Steinberg idempotent in $\overline{\mathcal{H}}_{0}$, we see from [SZ], Proposition 4.2 that the character $\bar{\lambda}_{0}$ which we obtained by restriction and reduction corresponds to a generalized Steinberg representation of the finite field group. This implies that the constituent $\pi$ of $V^{1+\mathfrak{P}_{1}}$ is the generalized Steinberg representation of $\overline{\mathfrak{A}}_{1}^{\times}$with cuspidal support $\tau=\sigma^{\otimes e^{\prime}}$

3.2 Proposition. The map $\mathcal{J L}_{A, D_{n}}: \mathcal{R}_{0}^{2}\left(D_{n}^{\times}\right) \rightarrow \mathcal{R}_{0}^{2}\left(A^{\times}\right)$is a bijection.

Proof based on AMT. The proof will be presented in several steps which we shall formulate as lemmas and corollaries. We shall compare characters $\Pi^{D_{n}} \in S_{\chi}^{D_{n}}$ and $\Pi^{A} \in S_{\chi}^{A}$ at very regular elements.

First we recall that very regular elements $x \in A^{\times}$are defined by the following properties:

(i) $F[x] \mid F$ is an unramified extension of degree $n$ in $A$,

(ii) $x$ is a unit of $F[x]$

(iii) the reduction $\bar{x}$ of $x$ modulo the prime ideal of $F[x]$ generates the residue field extension $k_{n} \mid k$.

If $x$ is very regular we can embed it into the multiplicative group of the standard maximal order: $x \in \mathfrak{A}_{1}^{\times} \subset A^{\times}$. Let $\varphi_{F, x} \in \mathfrak{o}_{F}[T]$ be the minimal polynomial of $x$ over $F$ and let $\bar{\varphi}_{F, x} \in k_{F}[T]$ be the reduction modulo $\mathfrak{p}_{F}$. Obviously for $\bar{x}=x \bmod \mathfrak{P}_{1}$ we have $\bar{\varphi}_{F, x}(\bar{x})=\overline{\varphi_{F, x}(x)}=0 \in \mathfrak{A}_{1} / \mathfrak{P}_{1}$. Therefore $\bar{\varphi}_{F, x^{\prime}}=\bar{\varphi}_{F, x}$ for all $x^{\prime} \in x\left(1+\mathfrak{P}_{1}\right)=x+\mathfrak{P}_{1}$, i.e. the whole coset consists of very regular elements.

3.3 Lemma. Let $x, x^{\prime} \in \mathfrak{A}_{1}^{\times}$be very regular. Then $\bar{\varphi}_{F, x^{\prime}}=\bar{\varphi}_{F, x}$ if and only if $y x^{\prime} y^{-1} \in x\left(1+\mathfrak{P}_{1}\right)$ for some $y \in\left\langle\varpi_{D}\right\rangle \cdot \mathfrak{A}_{1}^{\times}$.

Proof. It suffices to prove "only if". By Skolem/Noether, $\bar{\varphi}_{F, x^{\prime}}=\bar{\varphi}_{F, x}$ if and only if $\bar{x}, \bar{x}^{\prime} \in\left(\mathfrak{A}_{1} / \mathfrak{P}_{1}\right)^{\times}=G L_{m}\left(k_{D}\right)$ are conjugate up to an action of the Galois group $\operatorname{Gal}\left(k_{D} \mid k\right)$. Since conjugation by a prime element $\varpi_{D} \in D$ which normalizes the maximal unramified field extension $F_{d} \subset D$ generates $\operatorname{Gal}\left(k_{d} \mid k\right)$, there exists $y \in\left\langle\varpi_{D}\right\rangle \cdot \mathfrak{A}_{1}^{\times}$such that $\bar{y} \bar{x}^{\prime} \bar{y}^{-1}=\bar{x}$. But this pulls back to $y x^{\prime} y^{-1} \in x\left(1+\mathfrak{P}_{1}\right)$.

Let $\Pi^{A}=\mathcal{J L}_{A, D_{n}}\left(\Pi^{D_{n}}\right)$, where $\Pi^{D_{n}}$ is a level zero representation. Recall that the character $\Theta_{\Pi}$ of an admissible representation $\Pi$ of a p-adic reductive group $G$ is defined as a linear functional on the space of complex-valued, compactly supported, locally constant test functions defined on $G$. By Harish-Chandra's result ([HC], $§ 4$, Cor.) $\Theta_{\Pi}$ is represented by a locally constant function on the regular set of $G$.

For the following we use AMT:

3.4 Lemma. Let $x \in \mathfrak{A}_{1}^{\times}$be very regular. The character $\Theta_{\Pi^{A}}$ is represented on the coset $x\left(1+\mathfrak{P}_{1}\right)$ by a constant function.

Proof. Since the character $\Theta_{\Pi^{D_{n}}}$ is a level zero character, its value at any very regular element $x$ depends only on the reduction $\bar{x}$ and only on the reduction of 
the minimal polynomial $\bar{\varphi}_{F, x}$. By AMT the same is true for the values of $\Theta_{\Pi^{A}}$ at very regular $x \in \mathfrak{A}_{1}^{\times}$. From Lemma 3.3 it therefore follows that $\Theta_{\Pi^{A}}$ is constant on cosets $x\left(1+\mathfrak{P}_{1}\right)$ of very regular $x \in \mathfrak{A}_{1}^{\times}$.

3.5 Lemma. Let $x \in \mathfrak{A}_{1}^{\times}$be very regular. The character $\Theta_{\Pi^{A}}$ is represented on the coset $x\left(1+\mathfrak{P}_{1}\right)$ by the finite sum $\sum_{\theta} \theta$, where $\theta$ is summed over the irreducible level zero characters of $\mathfrak{A}_{1}^{\times}$which occur in $\left.\Pi^{A}\right|_{\mathfrak{A}_{1}^{\times}}$and each is counted once. Moreover, the two constant functions $\Theta_{\Pi^{A}}(u)$ and $\sum_{\theta} \theta(u)$ on $x\left(1+\mathfrak{P}_{1}\right)$ are identically equal.

Proof. For any locally constant test function $f$ supported in $\mathfrak{A}_{1}^{\times}$there is a congruence subgroup $1+\mathfrak{P}_{1}^{i_{f}}$ such that $f$ is bi-invariant with respect to $\left(1+\mathfrak{P}_{1}^{i_{f}}\right)$. Let $V$ be a representation space of $\Pi^{A}$ and for any $i>0$ let $V_{i} \subset V$ denote the subspace of $\left(1+\mathfrak{P}_{1}^{i}\right)$-invariant vectors. Then for any $i \geq i_{f}$ we may regard $\Pi^{A}(f)$ as an element of End $\left(V_{i}\right)$ and therefore

$$
\Theta_{\Pi^{A}}(f)=\operatorname{tr}\left(\Pi^{A}(f)\right)=\int_{\mathfrak{A}_{1}^{\times}} \Theta_{i}(u) f(u) d u
$$

where $\Theta_{i}(u)$ is the trace of the block of $\Pi^{A}(u)$ consisting of the matrix coefficients with respect to a basis of $V_{i}$. Then $\Theta_{i}=\sum_{j} m_{j} \theta_{j}$, where $\theta_{j}$ ranges over the finitely many characters of $\mathfrak{A}_{1}^{\times}$which are $\left(1+\mathfrak{P}_{1}^{i}\right)$-invariant and $m_{j} \geq 0$ denotes the multiplicity with which $\theta_{j}$ occurs in $\left.\Pi^{A}\right|_{\mathfrak{A}_{1}^{\times}}$, i.e. the multiplicity with which $\theta_{j}$ occurs in $V_{i}$. Now assume that $f$ has support in $x\left(1+\mathfrak{P}_{1}\right)$ and normalize the Haar measure on $A^{\times}$such that $\operatorname{vol}\left(1+\mathfrak{P}_{1}\right)=1$. Since the character $\Theta_{\Pi^{A}}$ is equal to a constant $\kappa$ on the set $x\left(1+\mathfrak{P}_{1}\right)$, we have

$$
\Theta_{\Pi^{A}}(f)=\int_{x\left(1+\mathfrak{P}_{1}\right)} \kappa f(u) d u=\int_{1+\mathfrak{P}_{1}} \kappa E(u) f\left(u^{-1} x\right) d u=\kappa(E * f)(x),
$$

where $E$ is the characteristic function of $1+\mathfrak{P}_{1}$. In particular, $E * E=E$, so we also have

$$
\Theta_{\Pi^{A}}(f)=\kappa(E * E) * f(x)=\kappa(E *(E * f))(x) .
$$

Thus, by (3.2) and (3.3), $\Theta_{\Pi^{A}}(f)=\Theta_{\Pi^{A}}(E * f)$ for all $f$ with support in $x\left(1+\mathfrak{P}_{1}\right)$. On the other hand, using (3.1) we also have

$$
\Theta_{\Pi^{A}}(f)=\int_{1+\mathfrak{P}_{1}} \Theta_{i}(u x)(E * f)(u x) d u=\int_{1+\mathfrak{P}_{1}}\left(E * \Theta_{i}\right)(u x) f(u x) d u .
$$

But $E * \theta$ is identically zero for any irreducible character $\theta$ of $\mathfrak{A}_{1}^{\times}$unless $\theta$ is a level zero character, in which case $E * \theta=\theta$. Therefore, from the representation of $\Theta_{i}$ as a finite sum of irreducible characters of $\mathfrak{A}_{1}^{\times}$we obtain $E * \Theta_{i}=\Theta_{1}$ for all $i \geq 1$. From this we see that

$$
\Theta_{\Pi^{A}}(f)=\int_{x\left(1+\mathfrak{P}_{1}\right)} \Theta_{1}(u) f(u) d u
$$

for all test functions $f$ supported on $x\left(1+\mathfrak{P}_{1}\right)$. Therefore, the function $\Theta_{1}$ represents the distribution $\Theta_{\Pi^{A}}$ on $x\left(1+\mathfrak{P}_{1}\right)$, which implies that $\Theta_{1}(u)=\Theta_{\Pi^{A}}(u)$ (as 
functions) for all $u \in x\left(1+\mathfrak{P}_{1}\right)$. From Lemma 3.1(i) we know that $\Theta_{1}$ is a sum of characters each occurring simply, hence the result.

Using [SZ], Theorem 1.1(ii) together with the explicit character formula for $\Theta_{\Pi^{D_{n}}}$, we see that there exists a very regular element $x \in D_{n}^{\times}$such that $\Theta_{\Pi^{D_{n}}}(x) \neq$ 0 . Therefore, we also see that $\Theta_{\Pi^{A}}(x)=\Theta_{\mathcal{J L}\left(\Pi^{D_{n}}\right)}(x) \neq 0$ (we identify regular elliptic conjugacy classes of $D_{n}^{\times}$and $A^{\times}$with the same minimal polynomials). We may conclude that $\left.\Pi^{A}\right|_{\mathfrak{A}_{1}^{\times}}$contains level zero components, so $\Pi^{A}$ is a level zero representation. Therefore $\mathcal{J L}_{A, D_{n}}$ gives an injection of $\mathcal{R}_{0}\left(D_{n}^{\times}\right)$into $\mathcal{R}_{0}^{2}\left(A^{\times}\right)$. This injection preserves the central character, i.e. $\mathcal{J L}_{A, D_{n}}$ takes $\mathcal{R}_{0}\left(D_{n}^{\times}, \omega\right)$ into $\mathcal{R}_{0}^{2}\left(A^{\times}, \omega\right)$, where $\omega$ is a fixed central character, i.e. a fixed character of $F^{\times}$. But on the other hand we know that for all $A$

$$
\mathcal{R}_{0}^{2}\left(A^{\times}, \omega\right)=\bigcup \mathcal{S}_{\chi}^{A}
$$

where $\chi \in X_{t}\left(F_{n}^{\times}\right)$runs over all characters such that $\chi_{F}=\omega$. Theorem 2.9(i) implies that $\mathcal{R}_{0}^{2}\left(D_{n}^{\times}, \omega\right)$ and $\mathcal{R}_{0}^{2}\left(A^{\times}, \omega\right)$ are finite sets of the same cardinality. Therefore, $\mathcal{J L}_{A, D_{n}}$ is surjective. Proposition 3.2 is proved.

3.6 Proposition. For $\Pi \in \mathcal{S}_{\chi}^{A}$ and $x \in A^{\times}$very regular

$$
\Theta_{\Pi^{A}}(x)=(-1)^{m-1} \sum_{\bar{\eta} \in[\bar{\chi}]} \bar{\eta}(\bar{x}) .
$$

Proof. Let $\Pi^{A} \in \mathcal{S}_{\bar{\chi}}^{A}$ and assume that $x \in A^{\times}$is very regular. Without loss of generality we may assume that $x \in \mathfrak{A}_{1}^{\times}$. Then we know from Lemma 3.3 combined with AMT that $\Theta_{\Pi^{A}}$ is constant on $x\left(1+\mathfrak{P}_{1}\right)$. By Lemma 3.4, $\Theta_{\Pi}=\Theta_{V^{1+\mathfrak{P}_{1}}}$ at very regular elements. We pass to $\overline{\mathfrak{A}}_{1}^{\times}$. Using 3.1(ii), we see that it is enough to compute the character at very regular elements by summing the characters of the generalized Steinberg components occurring in $V^{1+\mathfrak{P}_{1}}$. Thus, by 3.1(iii), we have a Galois orbit of length $(d, f)$ of GS representations corresponding to the $\mathfrak{S}_{e^{\prime}}$-orbits of length one in $V^{1+\mathfrak{P}_{e^{\prime}}}$, i.e. corresponding to the set of representations $\tau^{i}=\sigma^{\phi^{i} \otimes e^{\prime}}$, $0 \leq i<(d, f)$. Using [SZ], Theorem 6.1, we obtain

$$
\Theta_{\Pi^{A}}(x)=(-1)^{m-1} \sum_{\bar{\chi}^{\prime} \in \operatorname{Gal}\left(k_{d} \mid k\right) \cdot \bar{\chi}} \sum_{\bar{\eta} \in \operatorname{Gal}\left(k_{n} \mid k_{d}\right) \cdot \bar{\chi}^{\prime}} \bar{\eta}(\bar{x})=(-1)^{m-1} \sum_{\bar{\eta} \in \operatorname{Gal}\left(k_{n} \mid k\right) \cdot \bar{\chi}} \bar{\eta}(\bar{x}) .
$$

\section{§. Weak Explicit Matching Theorem.}

Now we prove our main result.

4.1 Weak Explicit Matching Theorem. Let $\chi \in X_{t}\left(F_{n}^{\times}\right)$, let $D_{n}$ be a central division algebra of index $n$ over $F$, and let $A$ be a central simple $F$-algebra of reduced degree $n$. Then $\mathcal{J L}_{A, D_{n}}\left(\mathcal{S}_{\chi}^{D_{n}}\right)=\mathcal{S}_{\chi}^{A}$ (cf 2.7), i.e. the Jacquet-Langlands map $\mathcal{J L}_{A, D_{n}}$ defines a bijection of $\mathcal{S}_{\chi}^{D_{n}}$ to $\mathcal{S}_{\chi}^{A}$ and of $\mathcal{S}_{\bar{\chi}}^{D_{n}}$ to $\mathcal{S}_{\bar{\chi}}^{A}$.

Proof. Let $\Pi^{A} \in \mathcal{S}_{\chi}^{A}$ and set $\Pi^{D_{n}}:=\mathcal{J L}_{D_{n}, A}\left(\Pi^{A}\right)$. The idea of the proof is to compare the characters $\Theta_{\Pi^{A}}$ and $\Theta_{\Pi^{D_{n}}}$ on the corresponding sets of very regular elements $x$. From (3.4) and (0.5) we obtain

$$
\Theta_{\Pi^{D_{n}}}(x)=\sum_{\bar{\eta} \in[\bar{\chi}]} \bar{\eta}(\bar{x})
$$


for $x$ very regular. On the other hand, Proposition 3.2 implies that $\Pi^{D_{n}}$ is a level zero representation of $D_{n}^{\times}$, and this implies that $\Pi^{D_{n}} \in \mathcal{S}_{\chi^{\prime}}^{D_{n}}$ for some $\chi^{\prime} \in X_{t}\left(F_{n}^{\times}\right)$. Applying (3.4) to $\Pi^{D_{n}} \in \mathcal{S}_{\chi^{\prime}}^{D_{n}}$ we obtain

$$
\Theta_{\Pi^{D_{n}}}(x)=\sum_{\bar{\eta}^{\prime} \in\left[\bar{\chi}^{\prime}\right]} \bar{\eta}^{\prime}(\bar{x}),
$$

again for all very regular elements $x$. Thus the character sums (4.1) and (4.2) are equal for very regular arguments. By [SZ] Theorem 1.1(i) this implies that $[\bar{\chi}]=\left[\bar{\chi}^{\prime}\right]$. Moreover, $\chi_{F}=\chi_{F}^{\prime}$ because $\Pi^{A}$ and $\Pi^{D_{n}}=\mathcal{J L}_{D_{n}, A}\left(\Pi^{A}\right)$ have the same central character (AMT). Therefore, since $\chi \in X_{t}\left(F_{n}^{\times}\right)$is determined by its restriction $\left.\chi\right|_{F^{\times}}$and its reduction $\bar{\chi},[\chi]=\left[\chi^{\prime}\right] \in\langle\phi\rangle \backslash X_{t}\left(F_{n}^{\times}\right)$. Thus $\mathcal{J L}_{D_{n}, A}$ maps $\mathcal{S}_{\chi}^{A}$ into $\mathcal{S}_{\chi}^{D_{n}}$. Since these finite sets contain the same number of elements (Proposition 2.8) and since, by AMT, $\mathcal{J L}_{D_{n}, A}$ is injective, we conclude that $\mathcal{J L}_{D_{n}, A}\left(\mathcal{S}_{\chi}^{A}\right)=\mathcal{S}_{\chi}^{D_{n}}$.

Remark. Since the inertial class of discrete series representations $\mathcal{S}_{\bar{\chi}}^{A}$ corresponds to a single Bernstein component $\Omega_{[\bar{\chi}]}$ and since associated supercuspidal supports $\left(M, \pi_{[\sigma]}^{\otimes e^{\prime}} \cdot \psi\right)$ also correspond to the same orbit $[\bar{\chi}]$, the "Weak Explicit Matching Theorem" implies that, at least up to unramified twist, the Jacquet-Langlands map commutes with parabolic induction (see $\S 0.5)$.

\section{$\S$ A. Appendix: Mod Center Hecke Algebras and $\mathcal{R}_{0}^{2}\left(A^{\times}\right)$.}

In order to justify assertions needed for the proofs of Propositions 2.4 and 2.8 we reformulate some parts of [BK1] and extend some of the results of [GSZ]. We review some of the connections between finite-dimensional mod center Hecke algebra modules and discrete series representations.

\section{$\S$ A.1 The Setting.}

We keep the notation and re-introduce some of the context of [GSZ]. From [GSZ], Part 4 we have the Hecke algebra $\mathcal{H}(r, z)$ of type $\tilde{A}_{r-1}(\mathrm{cf}$. [BK1](5.4)). Thus we have $\tilde{W}=W \rtimes\langle h\rangle$, an extension of the Coxeter group $(W, S)$, where $S$ is a set of cardinality $r$ and $\tilde{W}$ is an extension of $W$ by the infinite cyclic group $\langle h\rangle$. The group $\langle h\rangle$, acting on the normal subgroup $W$ by conjugation, stabilizes $S \subset W$ and cyclically permutes the elements of $S: h s_{i} h^{-1}=s_{i-1}(r-1 \geq i>0)$ and $h s_{0} h^{-1}=s_{r-1}$. The group $\tilde{W}$ is equipped with a length function $l$ which extends the length function on $W$ and satisfies $l\left(h^{i}\right)=0$ for all $i \in \mathbb{Z}$.

A1 Fact. For any $z \in \mathbb{C}^{\times}$the vector space $\mathbb{C}[\tilde{W}]$ has one and only one $\mathbb{C}$-algebra structure $\mathcal{H}(\tilde{W}, z)$ such that:

(i) $[s]^{2}=(z-1)[s]+z[1]$ for $s \in S$ and

(ii) $\left[w_{1}\right] \cdot\left[w_{2}\right]=\left[w_{1} w_{2}\right]$ whenever $l\left(w_{1} w_{2}\right)=l\left(w_{1}\right)+l\left(w_{2}\right)\left(w_{1}, w_{2} \in \tilde{W}\right)$.

\section{Remarks.}

(i) From (ii) we have $[h] \cdot[w]=[h w]$ and $[w] \cdot[h]=[w h]$ for all $w \in \tilde{W}$. The algebra

$$
\mathcal{H}(\tilde{W}, z)=\mathcal{H}(W, z) \otimes_{\mathbb{C}} \mathbb{C}[\langle[h]\rangle]
$$

is a twisted tensor product of the algebra for the Coxeter group $W$ with the group ring $\mathbb{C}[\langle[h]\rangle]$ :

$$
\left(\left[w_{1}\right] \otimes\left[h^{i}\right]\right)\left(\left[w_{2}\right] \otimes\left[h^{j}\right]\right)=\left[w_{1}\right]\left[h^{i} w_{2} h^{-i}\right] \otimes\left[h^{i+j}\right]
$$


for all $w_{1}, w_{2} \in W$ and $i, j \in \mathbb{Z}$.

(ii) The center of $\mathcal{H}(\tilde{W}, z)$ is the subalgebra $\mathcal{Z}:=\mathbb{C}\left[\left\langle\left[h^{r}\right]\right\rangle\right]$.

Now consider $A=M_{m}(D)$ and the distinguished pair $\left(F_{d}, \varpi\right)$, where $F_{d} \mid F$ is a maximal unramified extension of $F$ in $D$ and $\varpi$ denotes a prime element of $D$ which normalizes $F_{d}$; thus $\varpi^{d}=\varpi_{F}$, a prime element of $F$. The group $\tilde{W}_{A}$ denotes the extended relative affine Weyl group of $A^{\times}$; concretely, $\tilde{W}_{A}$ consists of all monomial matrices with entries which are either zero or powers of $\varpi$.

Let $\tau$ be the irreducible unitary representation of the principal order unit group $\mathfrak{A}_{r}^{\times}$which is lifted from the cuspidal representation $\sigma^{\otimes r}$ of $\overline{\mathfrak{A}}_{r}^{\times}=G L_{s}\left(k_{D}\right)^{\times r}(r s=$ $\left.m, k_{D}=k_{d}\right)$. Let $l$ be the cardinality of the Galois orbit $\operatorname{Gal}\left(k_{d} \mid k\right) \cdot \sigma$. Let $\tilde{W}$ denote the set of monomial $m \times m$ matrices of the form $w=T \otimes I_{s}$, where $T$ is an $r \times r$ monomial matrix with non-zero entries which are powers of $\varpi^{l}$ and $I_{s}$ denotes the $s \times s$ identity matrix. Let $W$ be the subgroup of $\tilde{W}$ consisting of all elements with reduced norm \pm 1 and let

$$
h:=\left[\begin{array}{ll} 
& I_{(r-1) s} \\
\varpi^{l} I_{s} &
\end{array} .\right.
$$

Then the support of the Hecke algebra $\mathcal{H}\left(A^{\times}, \mathfrak{A}_{r}^{\times}, \tau\right)$ of operator-valued $\tau$-spherical functions consists of the union of the set of double cosets $\mathfrak{A}_{r}^{\times} w \mathfrak{A}_{r}^{\times}$over $w \in \tilde{W}$, where $\tilde{W}=W \rtimes\langle h\rangle \subset \tilde{W}_{A}$ (see [GSZ], Proposition 2.1).

Slightly restating [GSZ](4.2), we have:

A2 Fact. Fix any non-trivial function $\mathcal{F} \in \mathcal{H}\left(A^{\times}, \mathfrak{A}_{r}^{\times}, \tau\right)$ which has support on $\mathfrak{A}_{r}^{\times} h \mathfrak{A}_{r}^{\times}$. Then there is a uniquely determined isomorphism of algebras

$$
\Phi_{\mathcal{F}}: \mathcal{H}\left(\tilde{W}, q^{d s}\right) \stackrel{\sim}{\longrightarrow} \mathcal{H}\left(A^{\times}, \mathfrak{A}_{r}^{\times}, \tau\right)
$$

such that:

(i) For all $w \in \tilde{W}$ the element $[w] \in \mathcal{H}\left(\tilde{W}, q^{d s}\right)$ is mapped to a function $\varphi_{w}$ which has support on $\mathfrak{A}_{r}^{\times} w \mathfrak{A}_{r}^{\times}$.

(ii) $\varphi_{h}=\Phi_{\mathcal{F}}([h])=\mathcal{F}$.

\section{$\S$ A2 Algebra Involutions and Isomorphisms of Hilbert Spaces.}

Let $\mathcal{W}$ be a representation space for the irreducible unitary representation $\tau$ and write $\langle,\rangle_{\mathcal{W}}$ for the inner product on $\mathcal{W}$. For $F \in \operatorname{End}_{\mathbb{C}}(\mathcal{W})$ let $F^{*}$ denote the adjoint of $F$ with respect to $\langle,\rangle_{\mathcal{W}}$ and define the involution $\star$ on $\mathcal{H}\left(A^{\times}, \mathfrak{A}_{r}^{\times}, \tau\right)$ by setting

$$
\varphi^{\star}(g):=\varphi\left(g^{-1}\right)^{*}
$$

for all $g \in A^{\times}, \varphi \in \mathcal{H}\left(A^{\times}, \mathfrak{A}_{r}^{\times}, \tau\right)$.

Clearly, the involution $\star$ defined by (12) sends a function with support on $\mathfrak{A}_{r}^{\times} w \mathfrak{A}_{r}^{\times}$ $(w \in \tilde{W})$ to a function with support on $\mathfrak{A}_{r}^{\times} w^{-1} \mathfrak{A}_{r}^{\times}$. Moreover, $(\lambda \varphi)^{\star}=\bar{\lambda} \varphi^{\star}$ for $\varphi \in \mathcal{H}\left(A^{\times}, \mathfrak{A}_{r}^{\times}, \tau\right), \lambda \in \mathbb{C}$, and $\left(\varphi_{1} * \varphi_{2}\right)^{\star}=\varphi_{2}^{\star} * \varphi_{1}^{\star}$ for all $\varphi_{1}, \varphi_{2} \in \mathcal{H}\left(A^{\times}, \mathfrak{A}_{r}^{\times}, \tau\right)$.

For the algebra $\mathcal{H}(\tilde{W}, z)$ we assume that $0<z \in \mathbb{R}$ and define the involution $\star$ such that $(\lambda[w])^{\star}:=\bar{\lambda}\left[w^{-1}\right](\lambda \in \mathbb{C}, w \in \tilde{W})$ and such that $(x+y)^{\star}=x^{\star}+y^{\star}$ $(x, y \in \mathcal{H}(\tilde{W}, z))$. Again we have an anti-commutative conjugate linear involution.

Next we define inner products on the algebras $\mathcal{H}\left(A^{\times}, \mathfrak{A}_{r}^{\times}, \tau\right)$ and $\mathcal{H}(\tilde{W}, z)$ : 
On $\mathcal{H}\left(A^{\times}, \mathfrak{A}_{r}^{\times}, \tau\right)$ we set

$$
\begin{aligned}
h\left(\varphi_{1}, \varphi_{2}\right): & =(\operatorname{dim} \tau)^{-1} \int_{A^{\times}} \operatorname{tr}_{\mathcal{W}}\left(\varphi_{1}(g) \cdot \varphi_{2}(g)^{*}\right) d g \\
& =(\operatorname{dim} \tau)^{-1} \operatorname{tr}_{\mathcal{W}}\left(\varphi_{1} * \varphi_{2}^{\star}\right)\left(1_{A^{\star}}\right) .
\end{aligned}
$$

On $\mathcal{H}(\tilde{W}, z)(0<z \in \mathbb{R})$ we define the inner product $\langle$,$\rangle by assuming that the set$ $\tilde{W}$ is an orthogonal basis for $\mathcal{H}(\tilde{W}, z)$ and setting $\langle[w],[w]\rangle=z^{l(w)}$. In particular, this implies that $\left\langle\left[h^{i}\right],\left[h^{i}\right]\right\rangle=1$ for any $i \in \mathbb{Z}$.

Let $\mathcal{F} \in \mathcal{H}\left(A^{\times}, \mathfrak{A}_{r}^{\times}, \tau\right)$ be a function supported on $\mathfrak{A}_{r}^{\times} h \mathfrak{A}_{r}^{\times}$. We know that $\mathcal{F} \in \mathcal{H}\left(A^{\times}, \mathfrak{A}_{r}^{\times}, \tau\right)^{\times}$. By Fact A2, there is a unique isomorphism

$$
\Phi_{\mathcal{F}}: \mathcal{H}\left(\tilde{W}, q^{d s}\right) \stackrel{\sim}{\longrightarrow} \mathcal{H}\left(A^{\times}, \mathfrak{A}_{r}^{\times}, \tau\right)
$$

such that $\Phi_{\mathcal{F}}([h])=\mathcal{F}$. Bushnell/Kutzko $([\mathrm{BK} 1](5.6 .16))$ call $\Phi_{\mathcal{F}}$ a unitary isomorphism if $\mathcal{F}^{\star}=\mathcal{F}^{-1}$.

On pp. 193-195 of [BK1], beginning at (5.6.17), Bushnell/Kutzko prove:

A3 Proposition. Assume that $\Phi_{\mathcal{F}}$ is a unitary homomorphism. Then:

(i) $\Phi_{\mathcal{F}}$ is a $\star$-homomorphism, i.e. $\Phi_{\mathcal{F}}\left(x^{\star}\right)=\Phi_{\mathcal{F}}(x)^{\star}$ for all $x \in \mathcal{H}\left(\tilde{W}, q^{d s}\right)$.

(ii) If the Haar measure on $A^{\times}$is normalized such that $\int_{\mathfrak{A}_{r}^{\times}} d x=1$, then $\Phi_{\mathcal{F}}$ is an isometry of pre-hilbert spaces, i.e. $h\left(\Phi_{\mathcal{F}}(x), \Phi_{\mathcal{F}}(y)\right)=\langle x, y\rangle$ for all $x, y \in \mathcal{H}\left(\tilde{W}, q^{d s}\right)$.

Proof. (i) Since $\Phi_{\mathcal{F}}$ is linear and both the involutions $\star$ are conjugate linear, it follows that, in order to show that $\Phi_{\mathcal{F}}$ is a $\star$-homomorphism, it is enough to show that $\Phi_{\mathcal{F}}\left([w]^{\star}\right)=\Phi_{\mathcal{F}}([w])^{\star}$ for all $w \in \tilde{W}$. Indeed, since $\left(\left[w_{1}\right] \cdot\left[w_{2}\right]\right)^{\star}=\left[w_{2}\right]^{\star} \cdot\left[w_{1}\right]^{\star}$ for all $w_{1}, w_{2} \in \tilde{W}$ and $\left(f_{1} * f_{2}\right)^{\star}=f_{2}^{\star} * f_{1}^{\star}$ for all $f_{1}, f_{2} \in \mathcal{H}\left(A^{\times}, \mathfrak{A}_{r}, \tau\right)$, it suffices to show that $\Phi_{\mathcal{F}}\left([s]^{\star}\right)=\Phi_{\mathcal{F}}([s])^{\star}$ for all $s \in S$ and that $\Phi_{\mathcal{F}}\left([h]^{\star}\right)=\Phi_{\mathcal{F}}([h])^{\star}$. Since $[s]^{\star}=[s]$, we must show that $\Phi_{\mathcal{F}}([s])^{\star}=\Phi_{\mathcal{F}}([s])$. Let $f:=f_{s}$ denote the obviously unique function in $\mathcal{H}\left(A^{\times}, \mathfrak{A}_{r}^{\times}, \tau\right)$ with support $\mathfrak{A}_{r}^{\times} s \mathfrak{A}_{r}^{\times}$such that $f * f=q^{d s} 1_{\mathcal{H}}+\left(q^{d s}-1\right) f$. Since $\Phi_{\mathcal{F}}([s])$ has support in $\mathfrak{A}_{r}^{\times} s \mathfrak{A}_{r}^{\times}$and $[s]^{2}=$ $q^{d s}[1]+\left(q^{d s}-1\right)[s]$, it follows that $\Phi_{\mathcal{F}}([s])=f$. Thus we must show that $f^{\star}=f$. On the one hand, we know that $\operatorname{tr}(f * f)(1)=q^{d s} \operatorname{dim}(\tau)>0$. Since $\left.s=s^{-1}\right)$, $f^{\star}$ also has support $\mathfrak{A}_{r}^{\times} s \mathfrak{A}_{r}^{\times}$. Thus, $f^{\star}=c f$ with $c \in \mathbb{C}^{\times}$; since $f^{\star \star}=f$ and $\star$ is conjugate linear, we have $|c|=1$. Since $f(x) f(x)^{*}$ is a positive operator on $\mathcal{W}$ for every $x \in \mathfrak{A}_{r}^{\times} s \mathfrak{A}_{r}^{\times}$, we see that

$$
\operatorname{tr}\left(f * f^{\star}\right)(1)=\operatorname{tr}\left(\int_{A^{\star}} f(x) f(x)^{*} d x\right)>0 .
$$

Therefore, $c=1$ and $f=f^{\star}$. Finally, if $\Phi_{\mathcal{F}}([h])=\mathcal{F}$, where $\mathcal{F}^{\star}=\mathcal{F}^{-1}$, then $\Phi_{\mathcal{F}}([h])^{\star}=\Phi_{\mathcal{F}}\left(\left[h^{-1}\right]\right)$, since $[h] *\left[h^{-1}\right]=1$ and $\Phi_{\mathcal{F}}$ is an isomorphism. Since $[h]^{\star}=\left[h^{-1}\right]$, we have shown that $\Phi_{\mathcal{F}}([h])^{\star}=\Phi_{\mathcal{F}}\left([h]^{\star}\right)$, which completes the proof of (i).

(ii) We begin with the following:

\section{A4 Lemma.}

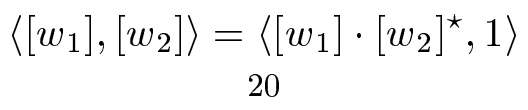


for all $w_{1}, w_{2} \in \tilde{W}$.

Proof. First we observe that any $w \in \tilde{W}$ can be written in the form $w^{\prime} h^{i}=$ $s_{i_{1}} \cdots s_{i_{l(w)}} h^{i}$, where $i \in \mathbb{Z}$ is unique and $w^{\prime}=s_{i_{1}} \cdots s_{i_{l(w)}}$ is a reduced expression for $w^{\prime} \in W$. If $w_{1}=w_{1}^{\prime} h^{i}$ and $w_{2}=w_{2}^{\prime} h^{i^{\prime}}$ with $i \neq i^{\prime}$, then both sides of (a.4) are zero, since $[w]^{\star}=\left[w^{-1}\right]$ and we may apply (a.1). Thus we need check only the case $i=i^{\prime}$ and it is clear that without loss of generality we may assume $i=0$, i.e. $w_{1}, w_{2} \in W$. Now we argue by induction on $l\left(w_{2}\right)$, assuming that $l\left(w_{1}\right)$ is arbitrary. The case $l\left(w_{2}\right)=0$, i.e. $w_{2}=1$ is trivial, so we assume $l\left(w_{2}\right)>0$ and that (a.4) is true for all $w_{1}$ and $w_{2}^{\prime}$ with $l\left(w_{2}^{\prime}\right)<l\left(w_{2}\right)$. Let $w_{2}=w_{2}^{\prime} s$ with $s \in S$ and $l\left(w_{2}^{\prime}\right)<l\left(w_{2}\right)$. By associativity in $\mathcal{H}(r, z)$ we have

$$
\left\langle\left[w_{1}\right] \cdot\left[w_{2}\right]^{\star}, 1\right\rangle=\left\langle\left[w_{1}\right] \cdot\left[w_{2}^{\prime} s\right]^{\star}, 1\right\rangle=\left\langle\left[w_{1}\right] \cdot\left[s w_{2}^{\prime-1}\right], 1\right\rangle=\left\langle\left(\left[w_{1}\right] \cdot[s]\right) \cdot\left[w_{2}^{\prime-1}\right], 1\right\rangle .
$$

There are two cases to consider: Assume $l\left(w_{1} s\right)>l\left(w_{1}\right)$. Then $\left[w_{1}\right] \cdot[s]=\left[w_{1} s\right]$. In this case, $\left\langle\left[w_{1}\right],\left[w_{2}\right]\right\rangle=0$, since $l\left(w_{2} s\right)=l\left(w_{2}^{\prime}\right)<l\left(w_{2}\right)$ implies that $w_{1} \neq w_{2}$. Moreover,

$$
\left\langle\left[w_{1} s\right] \cdot\left[w_{2}^{\prime}\right]^{\star}, 1\right\rangle=\left\langle\left[w_{1} s\right],\left[w_{2}^{\prime}\right]\right\rangle,
$$

since $l\left(w_{2}^{\prime}\right)\left\langle l\left(w_{2}\right)\right.$. Again it is clear that $\left\langle\left[w_{1} s\right],\left[w_{2}^{\prime}\right]\right\rangle=0$, since $w_{1} s \neq w_{2}^{\prime}$, i.e. $l\left(w_{1} s \cdot s\right)=l\left(w_{1}\right)<l\left(w_{1} s\right)$ and $l\left(w_{2}^{\prime} \cdot s\right)=l\left(w_{2}\right)>l\left(w_{2}^{\prime}\right)$. Thus (a.4) holds in the case that $l\left(w_{1} s\right)>l\left(w_{1}\right)$. Now assume that $w_{1} s=w_{1}^{\prime}$ and that $l\left(w_{1}^{\prime}\right)<l\left(w_{1}\right)$. In this case,

$$
\begin{aligned}
\left\langle\left(\left[w_{1}\right] \cdot[s]\right) \cdot\left[w_{2}^{\prime-1}\right], 1\right\rangle & =\left\langle\left[w_{1}^{\prime}\right](z[1]+(z-1)[s]) \cdot\left[w_{2}^{\prime-1}\right], 1\right\rangle \\
& =z\left\langle\left[w_{1}^{\prime}\right] \cdot\left[w_{2}^{\prime-1}\right], 1\right\rangle+(z-1)\left\langle\left[w_{1}^{\prime}\right] \cdot[s] \cdot\left[w_{2}^{\prime-1}\right], 1\right\rangle,
\end{aligned}
$$

using the distributivity and linearity of the multiplication in $\mathcal{H}(r, z)$. Now we have

$$
\left\langle\left[w_{1}^{\prime}\right] \cdot[s] \cdot\left[w_{2}^{\prime-1}\right], 1\right\rangle=\left\langle\left[w_{1}\right] \cdot\left[w_{2}^{\prime}\right]^{\star}, 1\right\rangle=\left\langle\left[w_{1}\right],\left[w_{2}^{\prime}\right]\right\rangle=0,
$$

since $w_{1} \neq w_{2}^{\prime}$ and we may use induction $\left(l\left(w_{2}^{\prime}\right)<l\left(w_{2}\right)\right)$. Also by induction we have

$$
z\left\langle\left[w_{1}^{\prime}\right] \cdot\left[w_{2}^{\prime-1}\right], 1\right\rangle=z\left\langle w_{1}^{\prime}, w_{2}^{\prime}\right\rangle=z \cdot z^{l\left(w_{2}^{\prime}\right)} \delta_{w_{1}^{\prime}, w_{2}^{\prime}}=z^{l\left(w_{2}\right)} \delta_{w_{1}, w_{2}},
$$

which completes the proof of the Lemma.

Now to complete the proof of Proposition A3 (ii): It is enough to prove the case $x=\left[w_{1}\right]$ and $y=\left[w_{2}\right]$ with $w_{1}, w_{2} \in \tilde{W}$ and to observe that then (ii) follows via linearity. Writing $\varphi_{w}:=\Phi_{\mathcal{F}}([w])$, we have

$$
(\operatorname{dim} \tau) h\left(\varphi_{w}, \varphi_{w}\right)=\operatorname{tr}_{\mathcal{W}}\left(\left(\varphi_{w} * \varphi_{w}^{\star}\right)(1)\right)=\operatorname{tr}_{\mathcal{W}}\left(\Phi_{\mathcal{F}}\left([w] \cdot[w]^{\star}\right)(1)\right) .
$$

From (a.4) and the orthogonality of the basis $\tilde{W}$ of $\mathcal{H}\left(\tilde{W}, q^{d s}\right)$ we obtain

$$
[w] \cdot[w]^{\star}=\langle[w],[w]\rangle 1+\sum_{w \neq 1} c_{w}[w] .
$$

Thus $\Phi_{\mathcal{F}}\left([w] \cdot[w]^{\star}\right)(1)=\langle[w],[w]\rangle \Phi_{\mathcal{F}}(1)(1)=\langle[w],[w]\rangle \mu\left(\mathfrak{A}_{r}^{\times}\right)^{-1} \tau(1)$. This gives

$$
h\left(\Phi_{\mathcal{F}}([w]), \Phi_{\mathcal{F}}([w])\right)=\langle[w],[w]\rangle \mu\left(\mathfrak{A}_{r}^{\times}\right)^{-1},
$$

which proves (ii), assuming that the measure $\mu\left(\mathfrak{A}_{r}^{\times}\right)=1$, and completes the proof of Proposition A3.

We must still prove that $\mathcal{F}$ may be chosen such that $\Phi_{\mathcal{F}}$ is unitary in the sense of Bushnell/Kutzko: 
A5 Lemma. There exists a function $\mathcal{F} \in \mathcal{H}\left(A^{\times}, \mathfrak{A}_{r}^{\times}, \tau\right)$ with support $\mathfrak{A}_{r}^{\times} h \mathfrak{A}_{r}^{\times}$such that $\mathcal{F}^{\star}=\mathcal{F}^{-1}$.

Proof. [GSZ], Lemma 1.1 implies that the function $\mathcal{F}$ has the value $\mathcal{F}(h)=J$, where $J \in \operatorname{Hom}_{\mathfrak{A}_{r}^{\times} \cap h^{-1} \mathfrak{A}_{r}^{\times} h}\left(\tau, \tau^{h}\right)$ is an intertwining operator. In [GSZ], Lemma 4.3 we saw that if $\tilde{\mathcal{F}} \in \mathcal{H}\left(A^{\times}, \mathfrak{A}_{r}^{\times}, \tau\right)$ has support $\mathfrak{A}_{r}^{\times} h^{-1} \mathfrak{A}_{r}^{\times}$and value $\tilde{\mathcal{F}}\left(h^{-1}\right)=J^{-1}$, then $\mathcal{F} * \tilde{\mathcal{F}}=\lambda 1_{\mathcal{H}}$, where $\lambda=\mu\left(\mathfrak{A}_{r}^{\times}\right) \mu\left(\mathfrak{A}_{r}^{\times} h \mathfrak{A}_{r}^{\times}\right)$. Thus if we replace $J$ by $J_{1}=\lambda^{-1 / 2} J$ as the value of $\mathcal{F}(h)$ and $J^{-1}$ by $J_{2}=\lambda^{-1 / 2} J^{-1}$ as the value of $\tilde{\mathcal{F}}\left(h^{-1}\right)$, then $\mathcal{F}^{-1}=\tilde{\mathcal{F}}$. Since $\mathcal{F}^{\star}\left(h^{-1}\right)=\mathcal{F}(h)^{*}=J_{1}^{*}$, it suffices to show that $J \in \operatorname{End}_{\mathbb{C}}(\mathcal{W})$ may be chosen to be a unitary operator, since in this case

$$
J_{1}^{*}=\lambda^{-1 / 2} J^{*}=\lambda^{-1 / 2} J^{-1}=J_{2}
$$

i.e., in this case, $\mathcal{F}^{\star}=\tilde{\mathcal{F}}=\mathcal{F}^{-1}$.

Let us show that $J$ can be chosen to be a unitary operator in $\operatorname{End}_{\mathbb{C}}(\mathcal{W})$. By the definition of $h$ (see (a.2)) we know that $h^{d r / l}=\varpi_{F} 1_{A^{\times}}$, a central element of $\tilde{W}$ ( $l$ is the length of the Galois orbit $\left.\operatorname{Gal}\left(k_{d} \mid k\right) \cdot \sigma.\right)$. The representation $\tau$ of $M\left(k_{D}\right)$ extends as a unitary representation to the group $M\left(k_{D}\right) \rtimes C$, where $C=\langle h\rangle \bmod$ $h^{d r / l}$, since $C$ is a finite cyclic group and $h^{d r / l}$ is central. Clearly, the generator $h$ of $C$ must under this extension map to an element of $\operatorname{Hom}_{\mathfrak{A}_{r}^{\times} \cap h^{-1} \mathfrak{A}_{r}^{\times} h}\left(\tau, \tau^{h}\right)=$ $\operatorname{Hom}_{M\left(k_{D}\right)}\left(\tau, \tau^{h}\right)$. This proves that $J$ can be chosen to be unitary and completes the proof of the Lemma.

\section{$\S$ A3 Discrete Series and Hecke Algebras with a Central Character.}

To reduce the description of discrete series in the general case to the unramified split case we reformulate some parts of $[\mathrm{BK} 1](6.1)$.

Let $\omega$ be a character of $\left\langle\varpi_{F}\right\rangle$ and let $\tau_{\omega}$ denote the extension of $\tau$ by $\omega$ to $\mathfrak{A}_{r}^{\times}\left\langle\varpi_{F}\right\rangle=\mathfrak{A}_{r}^{\times} F^{\times}$. We consider the projection maps:

$$
\mathcal{H}\left(\tilde{W}, q^{d s}\right) \longrightarrow \overline{\mathcal{H}}\left(\tilde{W}, q^{d s}\right):=\mathcal{H}\left(\tilde{W}, q^{d s}\right) /\left(\left[\varpi_{F}\right]-[1]\right)
$$

and

$$
\mathcal{H}\left(A^{\times}, \mathfrak{A}_{r}^{\times}, \tau\right) \longrightarrow \mathcal{H}\left(A^{\times}, \mathfrak{A}_{r}^{\times}\left\langle\varpi_{F}\right\rangle, \tau_{\omega}\right), \quad f \mapsto \bar{f}:=P f
$$

where $\operatorname{Pf}(x):=\sum_{i \in \mathbb{Z}} \omega\left(\varpi_{F}^{-i}\right) f\left(\varpi_{F}^{i} x\right)$. It is easy to see that the projection operator $P$ is a homomorphism of algebras, that the quotient algebra $\mathcal{H}\left(A^{\times}, \mathfrak{A}_{r}^{\times}\left\langle\varpi_{F}\right\rangle, \tau_{\omega}\right)$ has the structure of convolution algebra with respect to the product

$$
\left(\bar{f}_{1} * \bar{f}_{2}\right)(x)=\int_{A \times /\left\langle\varpi_{F}\right\rangle} \bar{f}_{1}(y) \bar{f}_{2}\left(y^{-1} x\right) d \dot{y} .
$$

The unit element is:

$$
\bar{e}(x)= \begin{cases}\mu\left(\mathfrak{A}_{r}^{\times}\right)^{-1} \tau_{\omega}(x) & \text { for } x \in \mathfrak{A}_{r}^{\times}\left\langle\varpi_{F}\right\rangle \\ 0 & \text { otherwise. }\end{cases}
$$

We fix the normalization $\mu\left(\mathfrak{A}_{r}^{\times}\right)=1$. 
A6 Lemma. Let $J \in \operatorname{End}_{\mathbb{C}}\left(\mathcal{W}_{\tau}\right)$ be constructed as in the second half of the proof of Lemma A5 and choose $\mathcal{F} \in \mathcal{H}\left(A^{\times}, \mathfrak{A}_{r}^{\times}, \tau\right)$ with support $\mathfrak{A}_{r}^{\times} h \mathfrak{A}_{r}^{\times}$such that

$$
\mathcal{F}(h):=\frac{1}{\sqrt{\mu}} J \zeta
$$

where $\mu=\mu\left(\mathfrak{A}_{r}^{\times} h \mathfrak{A}_{r}^{\times}\right)$and $\zeta$ is a root of unity chosen such that $\zeta^{d r / l}=\omega\left(\varpi_{F}\right)$. Then the isomorphism $\Phi_{\mathcal{F}}$ of Fact A2 induces an isomorphism

$$
\Phi_{\overline{\mathcal{F}}}: \overline{\mathcal{H}}\left(\tilde{W}, q^{d s}\right) \longrightarrow \mathcal{H}\left(A^{\times}, \mathfrak{A}_{r}^{\times}\left\langle\varpi_{F}\right\rangle, \tau_{\omega}\right),
$$

which implies the commutative diagram

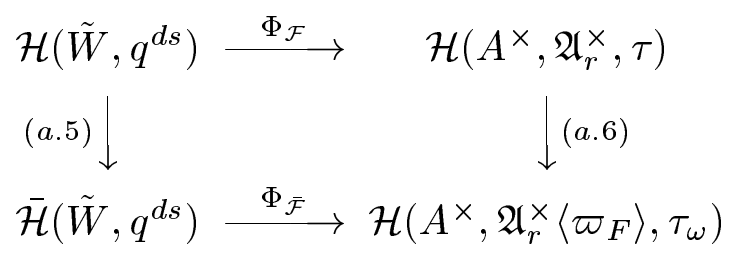

in which the horizontal arrows are the indicated isomorphisms and the numbers on the vertical arrows are the projection homomorphisms (a.5) and (a.6) defined previously.

Proof. Since $h^{r}=\varpi^{l} 1_{A^{\times}}$, an operator which normalizes $\mathfrak{A}_{r}^{\times}$, we have

$$
\mu\left(\mathfrak{A}_{r}^{\times} \cdot h^{r} \cdot \mathfrak{A}_{r}^{\times}\right)=\mu\left(h^{r} \mathfrak{A}_{r}^{\times}\right)=1 .
$$

Therefore, since $l$ is the length of the Galois orbit of $\sigma, \mathcal{F}^{r}\left(h^{r}\right)=J^{r} \zeta^{r}$ intertwines the representation $\tau$ of $\mathfrak{A}_{r}^{\times}$and, in particular, since by hypothesis $\zeta^{d r / l}=\omega\left(\varpi_{F}\right)$,

$$
\mathcal{F}^{d r / l}\left(\varpi_{F} 1_{A^{\times}}\right)=\mathcal{F}^{d r / l}\left(h^{d r / l}\right)=J^{d r / l} \zeta^{d r / l}=\omega\left(\varpi_{F}\right) 1_{\tau}=\bar{e}\left(\varpi_{F} 1_{A^{\times}}\right) .
$$

Thus $\overline{\mathcal{F}}^{d r / l}=\bar{e}$. We leave it to the reader to verify that the isomorphism of the affine Weyl group algebra $\left.\Phi_{\mathcal{F}}\right|_{\mathcal{H}\left(W, q^{d s}\right)}$ passes to the quotient and induces an isomorphism $\left.\Phi_{\overline{\mathcal{F}}}\right|_{\overline{\mathcal{H}}\left(W, q^{d s}\right)}$ such that the resulting subdiagram is commutative. In fact the vertical arrows for the affine Weyl group algebras are isomorphisms as well as the horizontal arrows.

We must show that the vertical algebra homomorphisms from the isomorphic cyclic group algebras $\mathbb{C}[\langle[h]\rangle]$ and $\mathbb{C}[\langle\mathcal{F}\rangle]$ involve the same polynomial relations, i.e. that

$$
\mathcal{H}\left(A^{\times}, \mathfrak{A}_{r}^{\times}, \tau\right) /\left(\mathcal{F}^{d r / l}-e\right) \cong \mathcal{H}\left(A^{\times}, \mathfrak{A}_{r}^{\times}\left\langle\varpi_{F}\right\rangle, \tau_{\omega}\right) .
$$

Since $[h] \mapsto \Phi_{\mathcal{F}}([h])=\mathcal{F}$ generates a homomorphism of cyclic groups, it suffices to show that no smaller power $\mathcal{F}^{\ell}$ of $\mathcal{F}$ maps to a scalar multiple of $\bar{e}$. For this we note that $\overline{\mathcal{F}}^{\ell}$ a root of the identity, i.e. a root of $\overline{\mathcal{F}}^{d r / l}=\bar{e}$, implies that $\ell \mid \frac{d r}{l}$. We therefore assume that $\ell \mid \frac{d r}{l}$. If $r \nmid \ell$, then the support of $\mathcal{F}^{\ell}$ is a double coset $\mathfrak{A}_{r}^{\times} h^{\ell} \mathfrak{A}_{r}^{\times}$such that $h^{\ell}$ does not normalize $\mathfrak{A}_{r}^{\times}$; in this case, $h^{\ell}$ cannot centralize the representation $\tau$, so $\mathcal{F}^{\ell}$ cannot be a multiple of the identity element $\bar{e}$. Assume that $r \mid \ell$. In this case the support of the function $\mathcal{F}^{\ell}$ is the double coset $\mathfrak{A}_{r}^{\times} h^{\ell}$ and 
$h^{\ell}=\varpi^{\ell l / r} 1_{A^{\times}} ;$since $r \mid \ell, h^{\ell}$ normalizes $\mathfrak{A}_{r}^{\times}$and the double coset supported at $h^{\ell}$ is the same as a one-sided coset. We see also that

$$
J^{\ell} \zeta^{\ell}=\mathcal{F}^{\ell}\left(h^{\ell}\right)=\mathcal{F}\left(\varpi^{\ell l / r} \cdot 1_{A^{\times}}\right) .
$$

To complete the proof of the Lemma it is sufficient to show that the operator $J$, which intertwines the representation $\tau$, cannot centralize $\tau$. In this case, $\mathcal{F}^{\ell}$ cannot be a scalar multiple of $\bar{e}$.

Let us show that $J^{\ell}$ has to be conjugate to a non-trivial permutation matrix, which implies that there exists $x \in \mathfrak{A}_{r}^{\times}$such that $J^{\ell} \tau(x) J^{-\ell} \neq \tau(x)$, since the commuting algebra of $\tau$ consists of only scalar matrices by Schur's Lemma. It is enough to show this for $\left.\tau\right|_{M(O)}$, since the intertwining of this irreducible restriction already determines $J^{\ell}$. We give details only for the case $r=1$, since the general case involves no additional ideas. Recall that $\tau$ is the lift of a cuspidal representation of $M\left(k_{D}\right)$ to $M(O)$. By results of S. I. Gelfand, quoted in [SZ], the restriction $\tau_{\mathcal{Q}\left(k_{d}\right)}$ is irreducible, where $\mathcal{Q}\left(k_{D}\right)$ denotes the Gelfand subgroup of $M\left(k_{D}\right)=G L_{m}\left(k_{D}\right)$. Moroever, the irreducible representation of $\mathcal{Q}\left(k_{D}\right)$ is independent of the choice of cuspidal representation and is simply $\operatorname{Ind}_{U_{0}\left(k_{d}\right)}^{\mathcal{Q}\left(k_{d}\right)} \mu$, where $\mu$ is any generic character of $U_{0}\left(k_{D}\right)$, where $U_{0}\left(k_{D}\right)$ denotes the upper triangular unipotent subgroup of $M\left(k_{D}\right)$. It follows that we may choose a basis for the representation space of $\tau$ which consists of generic vectors for conjugates of $U_{0}\left(k_{D}\right)$. The Galois group $\operatorname{Gal}\left(k_{D} \mid k\right)$ acts as a permutation group on this basis of $\mathcal{W}_{\tau}$. Since the set of generic vectors for $U_{0}\left(k_{D}\right)$ consists of a single orbit under the multiplicative group of diagonal matrices $T\left(k_{D}\right)$ acting by conjugation with only the center acting trivially, and the group $\operatorname{Gal}\left(k_{D} \mid k\right)$ acts on $T\left(k_{D}\right)$ mod the center with only the identity acting trivially, it follows that

$$
x \mapsto \tau\left(\varpi^{\ell / r} x \varpi^{-\ell / r}\right)=J^{\ell} \tau(x) J^{-\ell}=\tau(x)
$$

for all $x \in M(O)$ if and only if $\frac{d}{l} \mid \frac{\ell}{r}$, since no smaller power of $\varpi$ permutes the basis for $\mathcal{W}_{\tau}$ trivially. Thus only when $\frac{d r}{l} \mid \ell$ can $\overline{\mathcal{F}}^{\ell}$ be a multiple of $\bar{e}$. We conclude that $\mathcal{F} \mapsto \overline{\mathcal{F}}$ defines an isomorphism

$$
\mathbb{C}[\langle[h]\rangle] /\left(\left[h^{d r / l}\right]-[1]\right) \stackrel{\sim}{\longrightarrow} \mathbb{C}[\langle\overline{\mathcal{F}}\rangle] .
$$

The rest of the proof involves checking that the conjugation action of $\mathcal{F}$ on the affine part of the Hecke algebra $\mathcal{H}\left(A^{\times}, \mathfrak{A}_{r}^{\times}, \tau\right)$ factors through the projection $\mathcal{F} \mapsto \overline{\mathcal{F}}$ and this we leave to the reader.

As a special case of the foregoing, we consider the algebra $A^{\prime}:=M_{r}\left(F_{d s}\right)$, where the extension $F_{d s} \mid F$ is unramified of degree $d s$. In $A^{\prime \times}$, we have the Iwahori subgroup $\mathcal{I}^{\prime \times}:=\mathfrak{A}_{r}^{\prime \times}$ and for $\tau^{\prime}$ we take the trivial representation $1:=1_{\mathcal{I}^{\prime} \times}$. The support of the Hecke algebra $\mathcal{H}\left({A^{\prime}}^{\times}, \mathcal{I}^{\prime \times}, 1\right)$ is the group of monomial matrices $\tilde{W}_{A^{\prime}}$ with entries which are powers of $\varpi_{F}$. As before, we have the isomorphism

$$
\Phi_{1}: \mathcal{H}\left(\tilde{W}_{A^{\prime}}, q^{d s}\right) \longrightarrow \mathcal{H}\left(A^{\prime \times}, \mathcal{I}^{\prime \times}, 1\right),
$$

where $h^{\prime}:=\left[\begin{array}{cc}0_{r-1,1} & I_{r-1} \\ \varpi_{F} & 0_{1, r-1}\end{array}\right], \mathcal{F}^{\prime} \in \mathcal{H}\left(A^{\prime \times}, \mathcal{I}^{\prime \times}, 1\right)$ has support $\mathcal{I}^{\prime} h^{\prime} \mathcal{I}^{\prime}=\mathcal{I}^{\prime} h^{\prime}$ with $\mathcal{F}^{\prime}\left(h^{\prime}\right)=1$, and $\Phi_{1}\left(h^{\prime}\right)=\mathcal{F}^{\prime}$. (Note that $\mathcal{H}\left(A^{\prime \times}, \mathcal{I}^{\prime \times}, 1\right)$ is an algebra of scalarvalued functions, since " 1 " is one-dimensional. Put precisely, $\mathcal{H}\left(A^{\prime \times}, \mathcal{I}^{\prime \times}, 1\right)$ is 
the convolution algebra consisting of all compactly supported functions which are constant on $\mathcal{I}^{\prime}$ double cosets.)

Recall that a set of representatives for the $\mathfrak{A}_{r}^{\times}$double cosets lying in the support of the algebra $\mathcal{H}(\tau):=\mathcal{H}\left(A^{\times}, \mathfrak{A}_{r}^{\times}, \tau\right)$ may be chosen to be the set of all matrices of the form $\varpi^{l v} p \otimes I_{s}$, where $\varpi$ is the prime element of $D$ which was fixed earlier, $l$ is the length of the Galois orbit $\operatorname{Gal}\left(k_{D} \mid k\right) \cdot \sigma, v=\left(v_{1}, \ldots, v_{r}\right) \in \mathbb{Z}^{r}, \varpi^{l v}=$ $\operatorname{diag}\left(\varpi^{l v_{1}}, \ldots, \varpi^{l v_{r}}\right)$, and $p$ is an $r \times r$ permutation matrix. We note that the extended Coxeter system $\tilde{W}=(W, S) \rtimes\langle h\rangle$ is naturally isomorphic to the extended Coxeter system $\tilde{W}^{\prime}:=\left(W^{\prime}, S^{\prime}\right) \rtimes\left\langle h^{\prime}\right\rangle$ via the map

$$
\varpi^{l v} p \otimes I_{s} \longmapsto \varpi_{F}^{v} p
$$

$v$ and $p$ as above. It is important to observe that the mapping (a.7) sends $\varpi_{F} I_{m} \in$ $A^{\times}$to $\varpi_{F}^{d / l} I_{r} \in A^{\prime \times}$. We have the isomorphisms:

$$
\mathcal{H}(\tau) \stackrel{\Phi_{\mathcal{F}}}{\leftarrow} \mathcal{H}\left(\tilde{W}, q^{d s}\right) \cong \mathcal{H}\left(\tilde{W}^{\prime}, q^{d s}\right) \stackrel{\Phi_{1}}{\rightarrow} \mathcal{H}\left(A^{\prime \times}, \mathcal{I}^{\prime \times}, 1\right) .
$$

Using (a.7) we may regard these isomorphisms as support-preserving.

Next, passing to quotients and using (a.7) again we have

$$
\overline{\mathcal{H}}\left(\tilde{W}, q^{d s}\right) \cong \overline{\mathcal{H}}\left(\tilde{W}^{\prime}, q^{d s}\right)
$$

where $\overline{\mathcal{H}}\left(\tilde{W}^{\prime}, q^{d s}\right):=\mathcal{H}\left(\tilde{W}^{\prime}, q^{d s}\right) /\left(\left[\varpi_{F}^{d / l}\right]-[1]\right)$ Instead of (a.5) we now use the projection mapping

$$
\mathcal{H}\left(A^{\prime \times}, \mathcal{I}^{\prime \times}, 1\right) \longrightarrow \mathcal{H}\left(A^{\prime \times}, \mathcal{I}^{\prime \times}\left\langle\varpi_{F}^{d / l}\right\rangle, 1\right)
$$

which sends $f^{\prime} \in \mathcal{H}\left(A^{\prime \times}, \mathcal{I}^{\prime \times}, 1\right)$ to

$$
\bar{f}^{\prime}(x):=\sum_{i \in \mathbb{Z}} f^{\prime}\left(\varpi_{F}^{-i d / l} x\right) \in \mathcal{H}\left(A^{\prime \times}, \mathcal{I}^{\prime \times}\left\langle\varpi_{F}^{d / l}\right\rangle, 1\right)
$$

The projection mapping $f^{\prime} \mapsto \bar{f}^{\prime}$ is clearly an algebra homomorphism. From (a.8), (a.10), and Lemma A6 we deduce the diagram of isomorphisms

$$
\mathcal{H}\left(\tau_{\omega}\right) \stackrel{\Phi_{\overline{\mathcal{F}}}}{\leftarrow} \overline{\mathcal{H}}\left(\tilde{W}, q^{d s}\right) \cong \mathcal{H}\left(\tilde{W}^{\prime}, q^{d s}\right) \stackrel{\Phi_{\overline{1}}}{\rightarrow} \mathcal{H}\left(A^{\prime \times}, \mathcal{I}^{\prime \times}\left\langle\varpi_{F}^{d / l}\right\rangle, 1\right)
$$

in which $\mathcal{H}\left(\tau_{\omega}\right):=\mathcal{H}\left(A^{\times}, \mathfrak{A}_{r}^{\times}\left\langle\varpi_{F}\right\rangle, \tau_{\omega}\right)$. Since the maps $\Phi_{\mathcal{F}}$ and $\Phi_{1}$ of (a.8) are unitary according to Bushnell/Kutzko's definition (see above Proposition A3), (a.8) preserves the prehilbert structures on $\mathcal{H}(\tau)$ and $\mathcal{H}\left(A^{\prime \times}, \mathcal{I}^{\prime \times}, 1\right)$, respectively. To introduce prehilbert structures on the algebras of (a.11) it is therefore enough to treat any single quotient algebra and then transport the structure to the remaining algebras, using the equivalence of the norms in (a.8). It is enough to treat the case of $\mathcal{H}\left(\tilde{W}, q^{d s}\right)$. For $w \in \tilde{W}$, the image under the projection $[w] \mapsto \overline{[w]}$ is a function with support $\bar{w} \in \tilde{W} /\left\langle\varpi_{F}\right\rangle$. If $\overline{\left[w_{1}\right]}=\overline{\left[w_{2}\right]} \in \overline{\mathcal{H}}\left(\tilde{W}, q^{d s}\right)$, then it is clear that $\left\|w_{1}\right\|=\left\|w_{2}\right\|$. Therefore, we may use any set of representatives for $\tilde{W} /\left\langle\varpi_{F}\right\rangle$ to define the norms of the projections to an orthogonal basis for $\overline{\mathcal{H}}\left(\tilde{W}, q^{d s}\right)$, i.e. we set $\|[\bar{w}]\|=\|w\|$. Thus we may conclude that (a.11) induces isomorphisms 
between the completions of the various constituents which are compatible with module structures with respect to the algebras. We denote the completions of an algebra and its projection, respectively, by $\mathcal{H}^{2}$ and $\overline{\mathcal{H}}^{2}$.

We consider the categories $\mathcal{M}:=\mathcal{M}\left(A^{\times}, \varpi_{F}, \omega\right)$ and $\mathcal{M}^{\prime}:=\mathcal{M}\left(A^{\prime \times}, \varpi_{F}^{d / l}, 1\right)$ of smooth representations, where, in the first case, $\varpi_{F}$ acts via $\omega\left(\varpi_{F}\right)$ and, in the second, $\varpi_{F}^{d / l}$ acts trivially. These categories are closed under forming subquotients. Therefore, the pairs $\left(\mathfrak{A}_{r}^{\times}\left\langle\varpi_{F}\right\rangle, \tau_{\omega}\right)$ and $\left(\mathcal{I}^{\prime \times},\left\langle\varpi_{F}^{d / l}\right\rangle, 1\right)$ are types corresponding to subcategories of $\mathcal{M}$ and $\mathcal{M}^{\prime}$, respectively.

Simplifying our notation, we denote our pairs by $(K, \rho)$ and the corresponding Hecke algebras by $\mathcal{H}(\rho)$. Let $\mathcal{M}_{\rho}$ denote the subcategory of $\mathcal{M}$ or $\mathcal{M}^{\prime}$ which consists of all representations which are generated by their $\rho$-isotypic components. We have the equivalences

$$
\mathcal{M}_{\rho} \ni \Pi \longmapsto \operatorname{Hom}_{K}(\rho, \Pi) \in \mathcal{H}(\rho)^{\text {opp }}-\text { modules. }
$$

We may also interpret $\mathcal{M}, \mathcal{M}^{\prime}$ as the category of $\mathcal{H}$-modules, where $\mathcal{H}$ denotes the algebra of functions on $A^{\times}, A^{\prime \times}$ respectively, such that $f\left(\varpi_{F} g\right)=\omega\left(\varpi_{F}\right) f(g)$ and $f^{\prime}\left(\varpi_{F}^{d / l} g^{\prime}\right)=f^{\prime}\left(g^{\prime}\right)$, respectively, and where the support is relatively compact. We embed $\mathcal{H}$ in each case into the Hilbert space completion $\mathcal{H}^{2}$, where the condition of relative compactness is replaced by square integrability on $A^{\times} /\left\langle\varpi_{F}\right\rangle, A^{\prime \times} /\left\langle\varpi_{F}^{d / l}\right\rangle$ respectively. Now we refer to the arguments of [BK1](7.7.3)-(7.7.5) and apply them in the present context. We replace the notations $\mathcal{H}(G, \omega), \mathcal{H}^{2}(G, \omega), \mathcal{H}(G, \lambda \omega)$, and $\mathcal{H}^{2}(G, \lambda \omega)$ of [BK1] with the notations $\mathcal{H}, \mathcal{H}^{2}, \mathcal{H}(\rho)$, and $\mathcal{H}^{2}(\rho)$ (see (a.11), (a.12)), where $(K, \rho)$ are the types we are interested in. Then we may obtain the following formulation from $[\mathrm{BK} 1](7.7 .5)$. In the following we write $\mathcal{M}_{\rho}^{\text {finite }} \subset \mathcal{M}_{\rho}$ for the full subcategory consisting of representations with finite composition series.

A 7 Proposition. Under the equivalence (a.12) of categories a representation $\Pi \in$ $\mathcal{M}_{\rho}^{\text {finite }}$ is square integrable if and only if the $\mathcal{H}(\rho)^{\mathrm{opp}}$-module $\mathrm{Hom}_{K}(\rho, \Pi)$ can be embedded into $\mathcal{H}^{2}(\rho)$.

We wish to formulate a consequence of this assertion. Let $(K, \rho):=\left(\mathfrak{A}_{r}^{\times}\left\langle\varpi_{F}\right\rangle, \tau_{\omega}\right)$ and $\left(K^{\prime}, \rho^{\prime}\right):=\left(\mathcal{I}^{\prime \times}\left\langle\varpi_{F}^{d / l}\right\rangle, 1\right)$. Let $\mathcal{M}_{\rho} \subset \mathcal{M}$ and $\mathcal{M}_{\rho^{\prime}}^{\prime} \subset \mathcal{M}^{\prime}$ be the corresponding subcategories.

A8 Corollary. The isomorphisms of (a.11) induce a discrete-series preserving equivalence of the categories $\mathcal{M}_{\rho}^{\text {finite }} \rightarrow \mathcal{M}_{\rho^{\prime}}^{\prime \text { finite }}$.

Proof. Let $\Pi \in \mathcal{M}_{\rho}^{\text {finite }}$ and consider the $\mathcal{H}(\rho)$-module $\operatorname{Hom}_{K}(\rho, \Pi)$. Using (a.11) we may consider $\operatorname{Hom}_{K}(\rho, \Pi)$ as an $\mathcal{H}\left(\rho^{\prime}\right)$-module. Since $\left(K^{\prime}, \rho^{\prime}\right)$ is a type, we also obtain a representation $\Pi^{\prime} \in \mathcal{M}_{\rho^{\prime}}^{\prime}$ finite such that

$$
\operatorname{Hom}_{K^{\prime}}\left(\rho^{\prime}, \Pi^{\prime}\right)=\operatorname{Hom}_{K}(\rho, \Pi)
$$

as $\mathcal{H}\left(\rho^{\prime}\right)$-modules. By Proposition A7, $\Pi$ is discrete series if and only if $\operatorname{Hom}_{K}(\rho, \Pi)$ embeds into $\mathcal{H}^{2}(\rho)$. Since (a.11) implies that the isomorphism $\mathcal{H}(\rho) \cong \mathcal{H}\left(\rho^{\prime}\right)$ extends to a unitary isomorphism of $\mathcal{H}^{2}(\rho) \cong \mathcal{H}^{2}\left(\rho^{\prime}\right)$, it follows that $\operatorname{Hom}_{K}(\rho, \Pi)$ embeds in $\mathcal{H}^{2}(\rho)$ if and only if the isomorphic module Hom ${ }_{K^{\prime}}\left(\rho^{\prime}, \Pi^{\prime}\right)$ embeds in $\mathcal{H}^{2}\left(\rho^{\prime}\right)$. This means that $\Pi \in \mathcal{M}_{\rho}^{\text {finite }}$ is discrete series if and only if the same is true of $\Pi^{\prime} \in \mathcal{M}_{\rho^{\prime}}^{\prime}$ finite . 
A9 Proposition. If $\Pi \in \mathcal{M}_{\rho}^{\text {finite }}$ is an irreducible discrete series module, then $\operatorname{dim}_{\mathbb{C}}\left(\operatorname{Hom}_{K}(\rho, \Pi)\right)=1$, i.e. $\rho$ occurs in $\Pi$ with multiplicity one.

Proof. If $\Pi$ is irreducible and discrete series, then the same holds for the corresponding representation $\Pi^{\prime} \in \mathcal{M}_{\rho^{\prime}}^{\prime \text { finite }}$. But $A^{\prime \times}=G L_{r}\left(F_{d s}\right)$ and for this split group Bernstein/Zelevinsky have proved that the discrete series which occur in $\operatorname{Irr}(\Omega)$, where $\Omega$ is an arbitrary Bernstein component, occur as at most a single unitary inertial class of representations ([Ze], 9.3). Therefore, the only discrete series which occur in the unramified principal series of $G L_{r}\left(F_{d s}\right)$, i.e. in the set of irreducible discrete series representations associated to the Bernstein component of $G L_{r}\left(F_{d s}\right)$ for which $\left(K^{\prime}, \rho^{\prime}\right)$ is a type, have to be unramified twists of the Steinberg representation of $G L_{r}\left(F_{d s}\right)$. For the Steinberg representation $S t$ of an arbitrary p-adic reductive group $G$ it is known that the corresponding Iwahori bi-invariant matrix coefficient gives a scalar-valued homomorphism of the algebra $\mathcal{H}(G, \mathcal{I}, 1)$ (see [Bo], $\S 5$, where a more general result is given though only for semi-simple groups). This implies that $1_{\mathcal{I}}$ occurs simply in $\mathrm{St}_{\mathcal{I}}$ and obviously also the same will remain true for any unramified twist of $S t$. This implies that Hom $K_{K^{\prime}}\left(\rho^{\prime}, \Pi^{\prime}\right)$ has $\mathbb{C}$-dimension one as an $\mathcal{H}\left(\rho^{\prime}\right)$-module. From (a.13) we obtain the Proposition.

A10 Corollary. The number of irreducible discrete series representations $\Pi$ of $A^{\times}$which contain $\left(\mathfrak{A}_{r}^{\times}\left\langle\varpi_{F}\right\rangle, \tau_{\omega}\right)$ is $d r / l$.

Proof. It is enough to count the number of irreducible discrete series representations of $A^{\prime \times}$ which contain $\left(\mathcal{I}^{\prime \times}\left\langle\varpi_{F}^{d / l}\right\rangle, 1\right)$. As we have seen in the proof of Proposition A9, each of these representations is an unramified twist of the Steinberg representation $S t$ of $G L_{r}\left(F_{d s}\right)$. Since the central character of $S t$ is trivial, it follows that the central character of $S t \otimes \tilde{\chi}$, where $\chi$ is an unramified character and $\tilde{\chi}:=\chi \circ \operatorname{Nrd}_{A^{\prime} \mid F_{d s}}$, is $\chi^{r}$. In order to have a central character which is trivial on $\left\langle\varpi_{F}^{d / l}\right\rangle$ we therefore have to choose $\chi$ of $F^{\times}$such that $\chi^{r}$ is trivial on $\left\langle\varpi_{F}^{d / l}\right\rangle$. We see that there are $d r / l$ such possibilities and this implies the result. 


\section{REFERENCES}

[Ba] A. Badulescu, Correspondance entre $G L_{n}$ et ses formes intérieures en caractéristique positive, Thèse présentée pour obtenir le grade de Docteur en Sciences, Université de Paris-sud, Centre d'Orsay, 1999.

[BD] J. Bernstein, Rédigé par P. Deligne, Le "centre" de Bernstein, Représentations des groupes réductifs sur un corps local, Collection dirigée par Jean Dieudonné, Hermann, Paris, 1984, pp. 1-32.

[BR] J. Bernstein and C. Rumelhart, Lectures on Representations of Reductive p-adic Groups, Manuscript, 1996.

[Bo] A. Borel, Admissible Representations of a Semi-Simple Group over a Local Field with Vectors Fixed under an Iwahori Subgroup, Inventiones math. 35 (1976), 233-259.

[BK1] C. Bushnell and P. Kutzko, The Admissible Dual of GL(N) via Compact Open Subgroups, Annals of Math. Studies No. 129, Princeton U. Press, Princeton, NJ, 1993.

[BK2] C. Bushnell and P. Kutzko, Smooth Representations of Reductive p-adic Groups: Structure Theory via Types, Proc. London Math. Soc. 77 (1998), 582-634.

[DKV] P. Deligne, D. Kazhdan, and M.-F. Vigneras, Représentations des algèbres centrales simples p-adiques, Représentations des groupes réductifs sur un corps local, Collection dirigée par Jean Dieudonné, Hermann, Paris, 1984, pp. 33-117.

[DM] F. Digne and J. Michel, Representations of Finite Groups of Lie Type, London Mathematical Society Student Texts 21, Cambridge U. Press, Cambridge, UK, 1991.

[GSZ] M. Grabitz, A. Silberger, and E.-W. Zink, Level Zero Types and Hecke Algebras for Local Central Simple Algebras, Humboldt Universität pre-print (2000).

[Gr] J. Green, The characters of the finite general linear groups, Trans. Amer. Math. Soc. 80 (1955), 402-447.

[HC] Harish-Chandra, A Submersion Principle and Its Applications, Harish-Chandra Collected Papers, vol. IV, Springer, New York, NY, 1984, pp. 439-446.

[Ro] J. Rogawski, Representations of $G L(n)$ and division algebras over a p-adic field, Duke Math. J. 50 (1983), 161-169.

[Si] A. Silberger, Introduction to Harmonic Analysis on Reductive p-adic Groups, Math. Notes Princeton U. Press 23, Princeton U. Press, Princeton, NJ, 1979.

[SZ] A. Silberger and E.-W. Zink, The Characters of Generalized Steinberg Representations of Finite General Linear Groups on the Regular Elliptic Set, Trans. Amer. Math. Soc. 352 (2000), 3339-3356.

[Ze] A. Zelevinsky, Induced Representations of Reductive p-adic Groups II, Ann. scient. Éc. Norm. sup. 4e série 13 (1980), 165-210.

24 August 2000

Cleveland State University, Cleveland, Ohio 44115

E-mail address: silberger@math.csuohio.edu

Humboldt-Universität, Institut für Mathematik, Rudower Chaussee 25, 10099 Berlin, Germany

E-mail address: zink@mathematik.hu-berlin.de 